\title{
Brain neurometabolites differences in individuals with subjective cognitive decline plus: a quantitative single- and multi-voxel proton magnetic resonance spectroscopy study
}

\author{
Zhongxian Yang ${ }^{1,2,3}$, Xing Wan ${ }^{1,2}$, Xinzhu Zhao ${ }^{1,2}$, Yu Rong ${ }^{4}$, Yi Wu ${ }^{5}$, Zhen $\mathrm{Cao}^{3}$, Qiuxia Xie ${ }^{1,2}, \mathrm{Min} \mathrm{Luo}^{1,2}$, \\ Yubao Liu ${ }^{1,2}$ \\ ${ }^{1}$ Medical Imaging Center, Shenzhen Hospital, Southern Medical University, Shenzhen, China; ${ }^{2}$ The Third School of Clinical Medicine, Southern \\ Medical University, Guangzhou, China; ${ }^{3}$ Medical Imaging Center, the Second Affiliated Hospital, Medical College of Shantou University, Shantou, \\ China; ${ }^{4}$ Department of Neurology, the People's Hospital of Gaozhou City, Maoming, China; ${ }^{5}$ Department of Neurology, Shantou Central Hospital \\ and Affiliated Shantou Hospital of Sun Yat-sen University, Shantou, China
}

Contributions: (I) Conception and design: All authors; (II) Administrative support: Z Yang, Y Liu; (III) Provision of study materials or patients: Y Rong, Y Wu; (IV) Collection and assembly of data: Z Yang, Z Cao, Q Xie; (V) Data analysis and interpretation: X Wan, X Zhao, M Luo; (VI) Manuscript writing: All authors; (VII) Final approval of manuscript: All authors.

Correspondence to: Prof. Yubao Liu. Medical Imaging Center, Shenzhen Hospital, Southern Medical University, Shenzhen, Guangdong, China. Email: ybliu28@163.com.

Background: Subjective cognitive decline plus could be an extremely early phase of Alzheimer's disease; however, changes of $\mathrm{N}$-acetylaspartate, myoinositol, and $\mathrm{N}$-acetylaspartate/myoinositol is still unknown at this stage. This study aimed to explore brain neurometabolic alterations in patients with subjective cognitive decline plus using quantitative single-voxel and multi-voxel ${ }^{1} \mathrm{H}$-magnetic resonance spectroscopy.

Methods: A total of 91 participants were enrolled and underwent a GE 3.0-T magnetic resonance imaging, including 33 elderly controls, 27 patients with subjective cognitive decline plus, and 31 patients with amnestic mild cognitive impairment (MCI). Single-voxel and multi-voxel ${ }^{1} \mathrm{H}$-magnetic resonance spectroscopy were used to investigate the differences in neurometabolite levels among the three groups.

Results: Compared with elderly controls, patients with subjective cognitive decline plus showed significant decline in $\mathrm{N}$-acetylaspartate and $\mathrm{N}$-acetylaspartate/myoinositol values in multiple regions, and amnestic MCI participants demonstrated more significant decreased $\mathrm{N}$-acetylaspartate and N-acetylaspartate/ myoinositol levels in multiple regions. The combined concentrations of $\mathrm{N}$-acetylaspartate with myoinositol showed an excellent discrimination between those with subjective cognitive decline plus and elderly controls as compared to that obtained using $\mathrm{N}$-acetylaspartate/myoinositol ratios with the area under the receiver operating characteristic curve of 0.895 and 0.860 , respectively. Likewise, the combined area under the curve for differentiating patients with subjective cognitive decline plus from amnestic MCI was obtained using the combined levels of $\mathrm{N}$-acetylaspartate with myoinositol was 0.892 . This was also higher than the combined area under the curve of 0.836 obtained using N-acetylaspartate/myoinositol ratios. Moreover, $\mathrm{N}$-acetylaspartate levels in the left hippocampus and left posterior cingulate cortex (PCC) was positively related to the Auditory Verbal Learning Test delayed recall scores in patients with subjective cognitive decline plus, whereas only the $\mathrm{N}$-acetylaspartate/myoinositol ratio was positively related to this scale scores in the left hippocampus.

Conclusions: Quantitative single-voxel and multi-voxel ${ }^{1} \mathrm{H}$-magnetic resonance spectroscopy can provide valuable information to detect alterative brain neurometabolites characteristics in patients with subjective

\footnotetext{
^ ORCID: Yubao Liu, 0000-0002-6676-7832; Zhongxian Yang, 0000-0002-9389-663x.
} 
cognitive decline plus. $\mathrm{N}$-acetylaspartate concentrations may be used as one of the earliest neuroimaging markers at this stage, while $\mathrm{N}$-acetylaspartate/myoinositol ratio could be more suitable for monitoring Alzheimer's disease progression.

Keywords: Subjective cognitive decline plus; amnestic mild cognitive impairment; brain neurometabolites; magnetic resonance spectroscopy; neuroimaging marker

Submitted Nov 19, 2020. Accepted for publication Apr 23, 2021.

doi: 10.21037/qims-20-1254

View this article at: http://dx.doi.org/10.21037/qims-20-1254

\section{Introduction}

Subjective cognitive decline (SCD), refers to a selfexperienced decline in cognitive capacity without measurable cognitive impairment, is regarded as one of the earliest manifestations of the Alzheimer's disease (AD) continuum and exhibits a higher risk of developing into AD (1). It is well-known that SCD is pervasive in the elderly over 65 years of age and approximately $50 \%$ maintain cognitively stable (2). Since the cognitive function in SCD individuals maintain within normal range, it is hard to capture the subtle cognitive decline that patients may undergo before the onset of mild cognitive impairment (MCI) with the current neuropsychological evaluations. Mitchell et al. reported that SCD individuals were almost twice as likely to develop into MCI compared to normal elderly people (3). Previous studies also showed that patients with SCD present with gray matter atrophy $(4,5)$, white matter alterations (6), brain metabolism decline (7), high $\beta$-amyloid accumulation (8), and functional activity disruption (9). These findings revealed that $\mathrm{SCD}, \mathrm{MCI}$, and $\mathrm{AD}$ appeared to be the same spectrum of disease $(10,11)$. Despite the prediction of dementia by SCD, the proportion of progression of MCI/ $\mathrm{AD}$ is low on account of its characteristics of heterogeneity. SCD could be characterized as a memory decline and/or multiple cognitive domain impairment. In addition to the pathophysiological factors of $\mathrm{AD}$, SCD could be caused by many other factors (psychological and physiological factors, drug effect, and other neurological or medical diseases), for instance (12). If the SCD criteria are applied to this study, the heterogeneity should be ruled out since AD-related SCD should not be confused by the above-mentioned situations that affect subjective cognitive ability. Compared with SCD, SCD plus could be a higher risk of AD that prior to amnesiac aMCI (aMCI). According to the SCD Initiative, as an enrichment criterion, the SCD plus concept in a population-based SCD increases the likelihood of SCD indicating pathological change due to $\mathrm{AD}$ (13). It has a set of specific SCD features permitting addition and subtraction of items on progression of research. The features of SCD plus include subjective decline only confined to the area of memory cognition, complaints in regard to SCD (within the past 5 years), onset of SCD greater than or equal to 60 years, worry associated with SCD, the complainers feeling worse than the same age stages (memory loss), cognitive decline confirmed by an observer, and the existence of the apolipoprotein E 4 (APOE \&4) carriers and other biomarkers evidence for a potentially risk progression to $\mathrm{AD}$. Therefore, with higher sensitivity and specificity for development into aMCI/AD, SCD plus obviously increased the likelihood of preclinical AD in individuals with $\operatorname{SCD}(14,15)$.

Proton magnetic resonance spectroscopy $\left({ }^{1} \mathrm{H}-\mathrm{MRS}\right)$ is a valuable non-invasive tool that can be used for the detection of several neurometabolites, and understanding the underlying pathophysiological processes in brain disorders (16). Many brain metabolites, such as $\mathrm{N}$-acetylaspartate (NAA), cholinecontaining compounds (Cho), total creatine $(\mathrm{tCr})$, myoinositol $(\mathrm{mI})$, and glutamine and glutamate $(\mathrm{Glx})$ can be investigated. As the most abundant amino acids, NAA is synthesized in the mitochondria and located primarily in neural cells, axons, and dendrites. It usually has been interpreted as a marker of neuronal integrity and function based on the energy metabolism in neuronal mitochondria (17). As a maker of neuroglial cells, $\mathrm{mI}$ is mainly located in the glial cells and plays an important role as the brain osmoregulator (18). Cho represents a component of cell membranes, which plays an important role in maintaining their integrity and function. As part of the high-energy phosphate buffering system, $\mathrm{tCr}$ (the sum of creatine and phosphocreatine) is considered relatively constant in the neurons and glia of the brain. Hence, its concentration is used as a reference value $(19,20)$. Reduced NAA/Cr may reflect decreased neuronal integrity and raised $\mathrm{mI} / \mathrm{Cr}$ may reflect glial activation related 
to neuronal degeneration, while decreased $\mathrm{NAA} / \mathrm{mI}$ could be more suitable for monitoring $\mathrm{AD}$ progression $(21,22)$. They use metabolite ratios rather than absolute concentrations, which has been challenged since $\mathrm{tCr}$ could have abnormal levels $(23,24)$ and thus, it is hard to draw definite conclusions on which of the two metabolites in the ratio was responsible for the distinction. There are some reports of $\mathrm{tCr}$ values changing across the MCI-AD spectrum $(25,26)$. Thus, using the absolute metabolite concentrations is as an accepted method since the true metabolites' levels could be determined (27). As an established and practical technology, MRS may supply brain neurometabolic information for the early phase of $\mathrm{AD}(28,29)$. In addition, the underlying mechanism with respect to which neurometabolites altered first in SCD plus should be precisely elucidated. Most studies consistently showed that decreased NAA, Glx, NAA/tCr and $\mathrm{Glx} / \mathrm{tCr}$, along with increased $\mathrm{mI}$ and $\mathrm{mI} / \mathrm{tCr}$ were observed in different areas of the brain in MCI and/or AD (19,30-32). However, the findings with respect to Cho were less consistent $(21,26,30)$. These results indicated that MCI and/ or $\mathrm{AD}$ are not only caused by damage to a single brain region, but also result from alterations in several brain regions. Multi-voxel ${ }^{1} \mathrm{H}$-magnetic resonance spectroscopy $\left({ }^{1} \mathrm{H}-\mathrm{MVS}\right)$ is also named chemical shift imaging (CSI), which contains two-dimensional, three-dimensional CSI. In our previous studies (30,33-35), we documented absolute quantification in ${ }^{1} \mathrm{H}-\mathrm{MVS}$ performed in a series of different diseases, including studies with MCI/aMCI. However, as far as we know, no study has reported the changes in metabolites in patients with SCD plus, especially using a combination of single-voxel and multi-voxel MRS ( ${ }^{1} \mathrm{H}-\mathrm{SVS}$ and $\left.{ }^{1} \mathrm{H}-\mathrm{MVS}\right)$. Therefore, identification of neuroimaging markers of SCD plus is crucial for early detection, early diagnosis, early intervention, and reducing the burden of $\mathrm{AD}$-prone populations.

We hypothesized that ${ }^{1} \mathrm{H}-\mathrm{SVS}$ and ${ }^{1} \mathrm{H}-\mathrm{MVS}$ could be useful in the diagnosis of SCD plus in clinical practice. Moreover, we speculated that the alterations of metabolites of ${ }^{1} \mathrm{H}-\mathrm{SVS}$ and ${ }^{1} \mathrm{H}-\mathrm{MVS}$ in vulnerable areas of AD, which may reflect early neurochemical alterations, precede the clinical onset, and predict preclinical AD. In order to enhance our knowledge of the underlying course of $\mathrm{AD}$, promote early diagnosis and screening, provide guidance for clinical treatment, and understand the underlying pathophysiology of individuals at different stages of the disease, we aimed to explore and evaluate the absolute metabolite alterations in patients with SCD plus compared to those in aMCI and elderly controls using ${ }^{1} \mathrm{H}-\mathrm{SVS}$ and ${ }^{1} \mathrm{H}-\mathrm{MVS}$ by multiple brain region positioning. In addition, we also evaluated the changes in MRS-detected metabolites in correlation with key neuropsychological test scores.

\section{Methods}

\section{Human participants and neuropsychological testing}

A total of 96 participants from September 2017 to July 2019 were recruited in this study. Participants with SCD plus and aMCI were enrolled from the outpatient department of neurology and age- and sex-matched elderly controls (ECs) were recruited from the medical examination center. Five subjects were excluded due to MR contraindications (pacemaker claustrophobia and intracranial metallic foreign body) and insufficient image quality. The final sample consisted of 33 ECs participants, 27 SCD plus participants, and 31 aMCI participants. The study was conducted in accordance with the Declaration of Helsinki (as revised in 2013) and approved by the Ethics Committee of the second Affiliated Hospital of Shantou University Medical College. Written informed consent was obtained from all participants or his/her legal guardians before enrolment. The study was reviewed and approved by the Institutional Review Board of the Second Affiliated Hospital of Shantou University Medical College (Protocol ID: 2017-10), and all procedures were conducted following the 1964 Helsinki declaration and its later amendments. Each participant provided demographic and clinical data and underwent neuropsychological assessments by two experienced neurologists. All participants underwent a battery of standardized neuropsychological assessments (including cognitive functioning in the area of memory, attention, executive functioning, and language). Psychological tests were carried out the day before MRI acquisition. These psychological tests included the Mini-Mental State Examination Scale (MMSE) (36), the Montreal Cognitive Assessment Scale (MoCA, Beijing version) (37), assessment on the Clinical Dementia Rating Scale (CDR) (38), global deterioration scale (GDS) (39), the Auditory Verbal Learning Test [AVLT, Chinese Huashan version, including three subtests: AVLT-immediate recall scores, AVLTdelayed recall scores, and AVLT-recognition scores] (40), an activities of daily living assessment (ADL) (41), the Hachinski Ischemic Scale (HIS) (42), and the Hamilton Depression Rating Scale (HAMD) (43).

SCD plus participants were enrolled according to the criteria proposed by SCD-I (13). Psychological tests that needed to be met were performance within normal range 

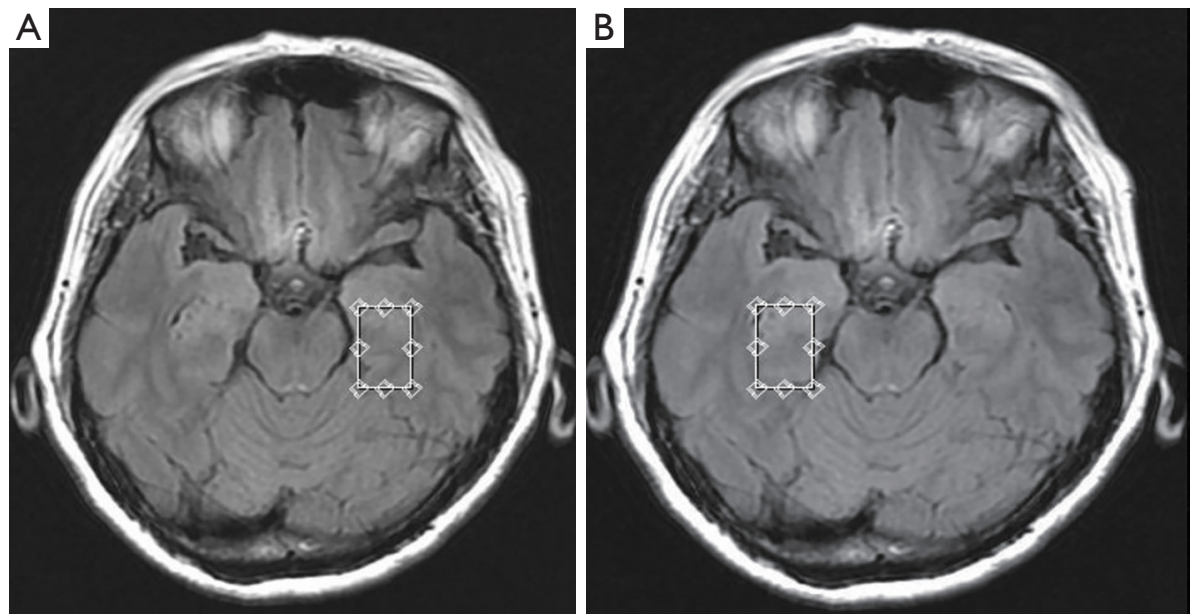

Figure 1 Representative location of the volume of interests set on bilateral hippocampus (A and B).

of the MoCA, MMSE, and ADL score after sex-, age-, and education adjustment, $\mathrm{CDR}$ score $=0$, GDS score $=2$, HIS score $<4$, and HAMD score $<7$. aMCI participants met the following criteria by Petersen et al. (44), including (I) memory decline confirmed by an informant or clinical judgment, (II) objective memory decline that must belong to the type of episodic memory, decided by neurologists' judgment on the basis of neuropsychological assessments, (III) CDR score $=0.5$, GDS score $=3$, HIS score $<4$, HAMD score $<7$, and ADL score $<22$, and (IV) did not meet the criteria by National Institute of Neurological and Communicative Diseases and Stroke-Alzheimer's Disease and Related Disorders Association. Inclusion criteria of ECs were as follows: no subjective cognitive complaints, no physical/psychiatric/neurological disorders, no abnormalities in routine brain MR imaging (including physiological brain atrophy), and no abnormalities on the neuropsychological measurement. The exclusion criteria were: (I) HIS score $>4$, (II) HAMD score $>24$, (III) white matter injury (multiple sclerosis, tuberous sclerosis, encephalitis, cranial arteritis, epilepsy, trauma, and tumor) (IV) dementia ( $\mathrm{AD}$, vascular dementia, frontotemporal dementia, Lewy body dementia), (V) Moyamoya disease, Parkinson's disease, aphasia, hepatic encephalopathy, intracranial hemorrhage, and systemic diseases, and (VI) history of alcohol dependence and other drug abuse, serious medical disease (liver and kidney dysfunction, heart and respiratory failure, chronic electrolyte disturbance, heavy metal poisoning), mental illness (depression, anxiety, bipolar disorder, schizophrenia); (VII) contraindications for MRI examination.
Conventional magnetic resonance imaging (MRI), MRS data acquisition and post-processing for metabolite quantification

Conventional MRI scans were obtained by a standard 8-channel head coil using a 3.0 T GE MRI Systems (Signa HDx Twin speed; GE Medical, Milwaukee, Wisconsin, USA). Host scanning was then changed from the clinical mode to research mode. Under this mode, axial T2 fluid attenuated inversion recovery images were used for performing spectroscopic acquisition. To ensure the same locations for each participant, the volume of interests (VOIs) for the ${ }^{1} \mathrm{H}-\mathrm{MRS}$ spectra were assigned to a total of 16 brain regions, as shown in Figure 1A,B and Figure 2 including the bilateral hippocampus (Hip), precuneus (Pr), posterior cingulate cortex (PCC), white matter of occipital lobe (OLWM), dorsal thalamus (DT), lenticular nucleus (LN), caput nuclei caudati (CNC), and white matter of frontal lobe (FLWM) for further metabolite quantification. Since each participant's head size differs, the brain structure is also slightly different. To minimize the impact of cerebrospinal fluid (CSF) contamination, skull bone and air on the metabolite concentration, the VOI acquisitions were determined based on the anatomical landmarks of each participant. Owing to poor shimming on the basicranial level due to the tissue-air interface near the petrous bone, a fully automated point-resolved spectroscopy (PRESS, Probe-P) pulse sequence was used to obtain single-voxel proton MRS data $\left({ }^{1} \mathrm{H}-\mathrm{SVS}\right.$, TE/TR $=35 / 1,500 \mathrm{~ms}$, NEX: 4, phase $\times$ frequency: $1 \times 1$ ) for the bilateral Hip. The SVS VOIs were average in size $3.00 \mathrm{~cm}^{3}[1.0 \mathrm{~cm}$ (width) $\times 2.0 \mathrm{~cm}$ 


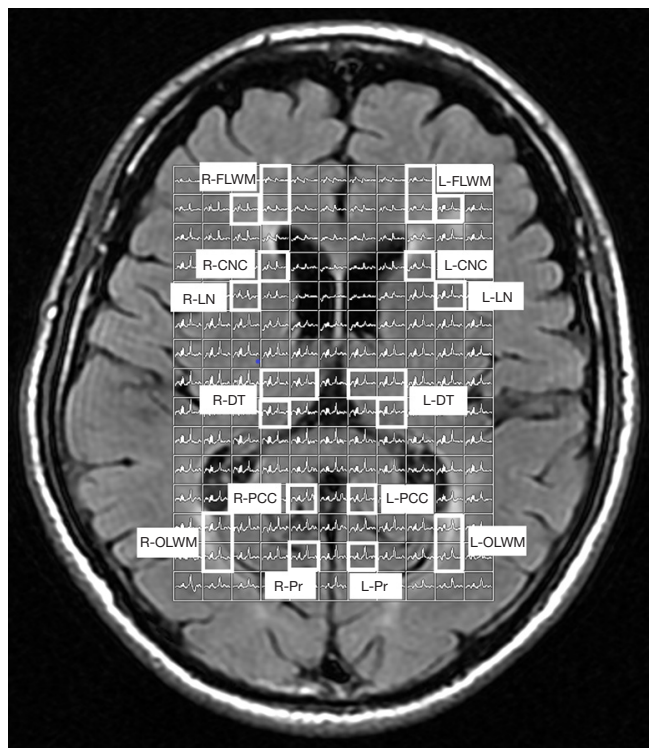

Figure 2 Representative location of the volume of interests set on axial view of basal ganglia slice for multivoxel $1 \mathrm{H}$-magnetic resonance spectroscopy. L-Pr, left precuneus; R-Pr, right precuneus; L-OLWM, left white matter of occipital lobe; R-OLWM, right white matter of occipital lobe; L-PCC, left posterior cingulate cortex; R-PCC, right posterior cingulate cortex; L-DT, left dorsal thalamus; R-DT, right dorsal thalamus; L-LN, left lenticular nucleus; R-LN, right lenticular nucleus; L-CNC, left caput nuclei caudati; R-CNC, right caput nuclei caudati; L-FLWM, left white matter of frontal lobe; R-FLWM, right white matter of frontal lobe.

(length) $\times 1.5 \mathrm{~cm}$ (thickness)]. Two-dimensional multivoxel proton MRS with PRESS pulse sequence (FOV: $16.0 \mathrm{~cm} \times 16.0 \mathrm{~cm} ; \mathrm{TE} / \mathrm{TR}=35 / 1,500 \mathrm{~ms}$, NEX: 1 , phase $\times$ frequency: $18 \times 18$ ) was performed at the basal ganglia level to acquire the multi-voxel MRS data. The VOIs of the multi-voxel section was about $8.0 \mathrm{~cm}$ (left to right) $\times$ $10.0 \mathrm{~cm}$ (anterior to posterior) $\times 1.5 \mathrm{~cm}$ (thickness). Thus, every single average VOI was $1.19 \mathrm{~cm}^{3}$ [(FOV: $16.0 \mathrm{~cm} \times$ $16.0 \mathrm{~cm} /$ phase, frequency: $18 \times 18) \times 1.5 \mathrm{~cm}]$. The VOI size and signal-to-noise ratio $(\mathrm{S} / \mathrm{N})$ in the left and right brain hemispheres of the three groups are shown in Table S1 and Table S2. Automatic prescan procedures such as calibration, shimming, and chemical shift selective water suppression were optimized as a concentration reference for the VOIs. The acquisition time of the ${ }^{1} \mathrm{H}-\mathrm{SVS}$ on each side of Hip were 3 minutes 42 seconds and the acquisition time of the ${ }^{1} \mathrm{H}-\mathrm{MVS}$ on basal ganglia slice were 8 minutes 12 seconds.

The raw data (P files, Supplementary file 1) were created by the host and were acquired from the dedicated workstation for Windows 4.6 (ADW 4.6, GE Medical, Rue de la Minière, France). All P files were subsequently imported to UNIX system (Red Hat Enterprise of Linux 8.1 version) to process the MR spectroscopic data. The data was voxel-shifted to align the CSI grid using the NAA VOI. As reported in our previous work (30,33-35), the data first underwent Fourier transformation and was subsequently processed using the software Spectroscopy Analysis by General Electric (SAGE version 7.0), which included automatic phase and frequency correction, line broadening, and zero-filling. The data were then entered into the Linear Combination of Model (LCModel version 6.3.1L, Stephen Provencher, Inc., Oakville, ON, Canada) for metabolite quantification. We used a standard detectable phantom concentration $(12.5 \mathrm{mmol} / \mathrm{L} \mathrm{NAA}, 3.0 \mathrm{mmol} / \mathrm{L}$ choline chloride, $10.0 \mathrm{mmol} / \mathrm{L}$ creatine hydrate, $5 \mathrm{mmol} / \mathrm{L}$ lactate, plus $0.1 \%$ sodium azide, $0.1 \%$ magnavis, and $50 \mathrm{mmol} / \mathrm{L}$ sodium hydroxide) as an outer reference to calibrate the concentrations of metabolite as well. The absolute concentrations of these metabolites (NAA, tCr, Cho, and $\mathrm{mI} ; \mathrm{mmol} / \mathrm{L}$ ) were then calculated from the VOI regions (See Supplementary file 2). The MVS of the metabolic profile were post-processed using the ReadyView software in the ADW 4.6 environment. SVS with full width at half maximum (FWHM) $\leq 7 \mathrm{~Hz}$ and MVS with FWHM $\leq 15 \mathrm{~Hz}$ were used for quality control. The quantifications of the metabolite concentrations were assessed using Cramér-Rao lower bounds (CRLBs). Metabolites quantified with CRLBs $>50 \%$ were classified as detected, and metabolites quantified with standard deviations CRLBs that did not exceed a threshold of $20 \%$ were considered for further analysis.

\section{Statistical analyses}

Statistical analyses were conducted by the SPSS statistics version 25.0 (SPSS Inc., Chicago, III, USA) and GraphPad Prism (Inc. Prism Version 8.3.0, USA). All comparisons of the means from the demographic data (such as age, sex) were performed using the Mann-Whitney $U$ test. Oneway analyses of variance (ANOVA) with Bonferroni's correction for post hoc (Bonferroni test) comparisons were used to assess neuropsychological tests among the three groups. As for metabolite concentrations, group differences were analyzed among the three groups by multivariate ANOVA using VOIs $(\times 16)$ and metabolites $(\times 4)$ as within and between factors. Bonferroni correction was used to perform post-hoc multiple pairwise comparisons among 
Table 1 The results of demographic features and key neuropsychological scores

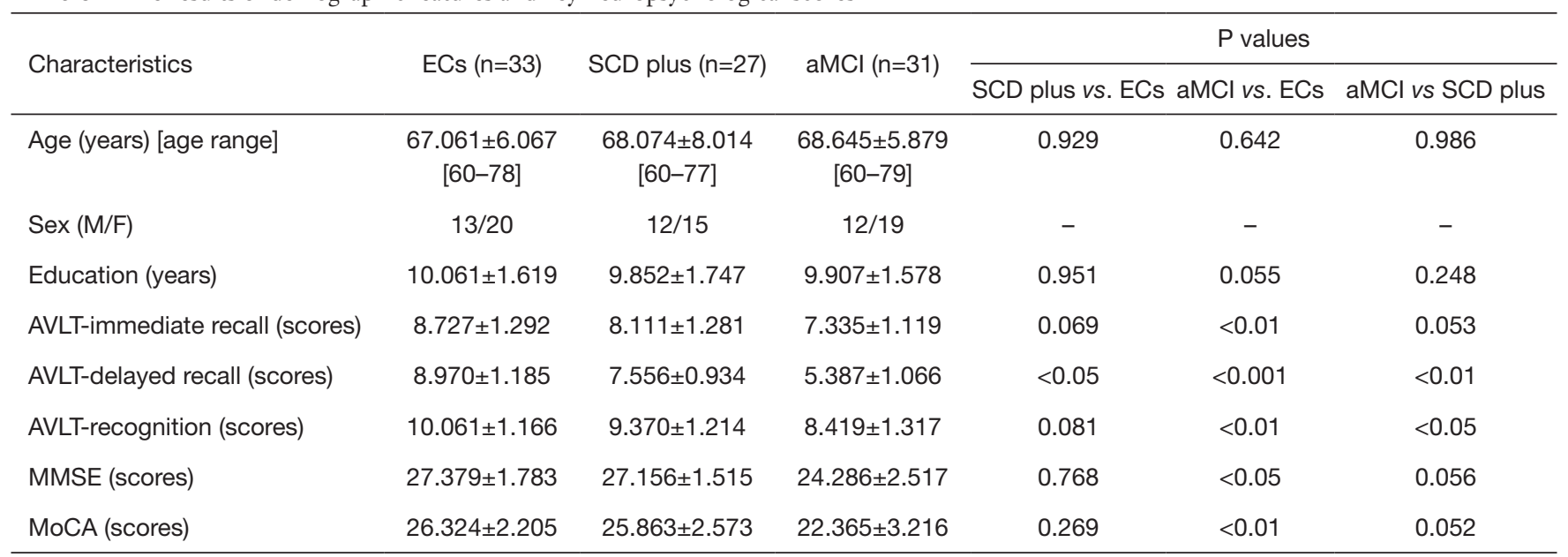

ECs, elderly controls; SCD plus, subjective cognitive decline plus; aMCI, amnestic mild cognitive impairment; M, male; F, female; AVLT, Auditory Verbal Learning Test; MMSE, Mini-Mental State Exam; MoCA, Montreal cognitive assessment.

the three groups. Nonparametric tests were used when the data was non-normal distribution. Pearson's correlation analysis controlling for sex, age, and length of education as covariates was performed to assess the relationship between the concentration of metabolites and the key neuropsychological scores for SCD plus participants. Heat maps were generated for the metabolites of interest by the $\mathrm{R}$ software (version 4.0.3) to a get an overview of the differences among the three groups. Furthermore, receiver operating characteristic (ROC) curve analysis was conducted to assess diagnostic accuracy of various neurometabolites. The area under the ROC curve (AUROC) was conducted to determine the predictive accuracy of the image metrics in distinguishing SCD plus from ECs or aMCI participants. In order to better distinguish the different groups and supply useful information to clinicians, combined AUC values were further acquired using binary logistic regression. Random forest (RF) was used to determine whether predictors load for the ROC curves and the model had problem of overfitting. All data are expressed as mean $\pm \mathrm{SD}$, and the significance threshold was set at $\mathrm{P}<0.05$.

\section{Results}

\section{Demographic and key neuropsychological scores results}

The demographic data of the subjects and key neuropsychological scores are summarized in Table 1. Only AVLT delayed recall (AVLT-De) scores between the ECs and SCD plus participants were significant. SCD plus participants showed significantly decreased AVLT-De and AVLT-recognition scores compared to aMCI participants. aMCI group showed significantly lower in all subitems of AVLT scores (AVLT-immediate recall scores, AVLTDe scores, and AVLT-recognition scores) than ECs group. In addition, only MoCA scores and MMSE scores were observed lower in aMCI compared with ECs participants. No significant differences were observed in terms of age, sex, and education among the participants.

\section{Differences in ${ }^{1} H$-MRS neurometabolites in participants with ECs, SCD plus, and aMCI}

The results of VOIs and S/N showed relatively stable performance (Tables S1,S2). Therefore, all measurements were regarded as reliable, and metabolite concentrations values were used for subsequent statistical analyses. NAA, $\mathrm{mI}$, Cho, $\mathrm{tCr}$ and $\mathrm{NAA} / \mathrm{mI}$ ratios were acquired in bilateral regions of the Hip, PCC, Pr, OLWM, FLWM, DT, LN, and CNC in three groups. Tables 2,3 show the differences in brain metabolite values from the left VOIs among the three groups. Tables 4,5 show the differences in brain metabolite levels from the right VOIs among the three groups. Figure S1A,B,C and Figure S2A,B,C show representative singlevoxel MRS in left and right Hip, respectively.

Compared with ECs group, SCD plus individuals were observed to have significantly reduced NAA levels in the left Hip, left PCC, left Pr and left CNC. mI value was only found elevated in the right Hip. The NAA/mI ratio 
Table 2 Concentration of the metabolites (unit, $\mathrm{mmol} / \mathrm{L}$ ) and NAA/mI ratios in the left volume of interests (VOIs) of ECs, SCD plus, and aMCI participants

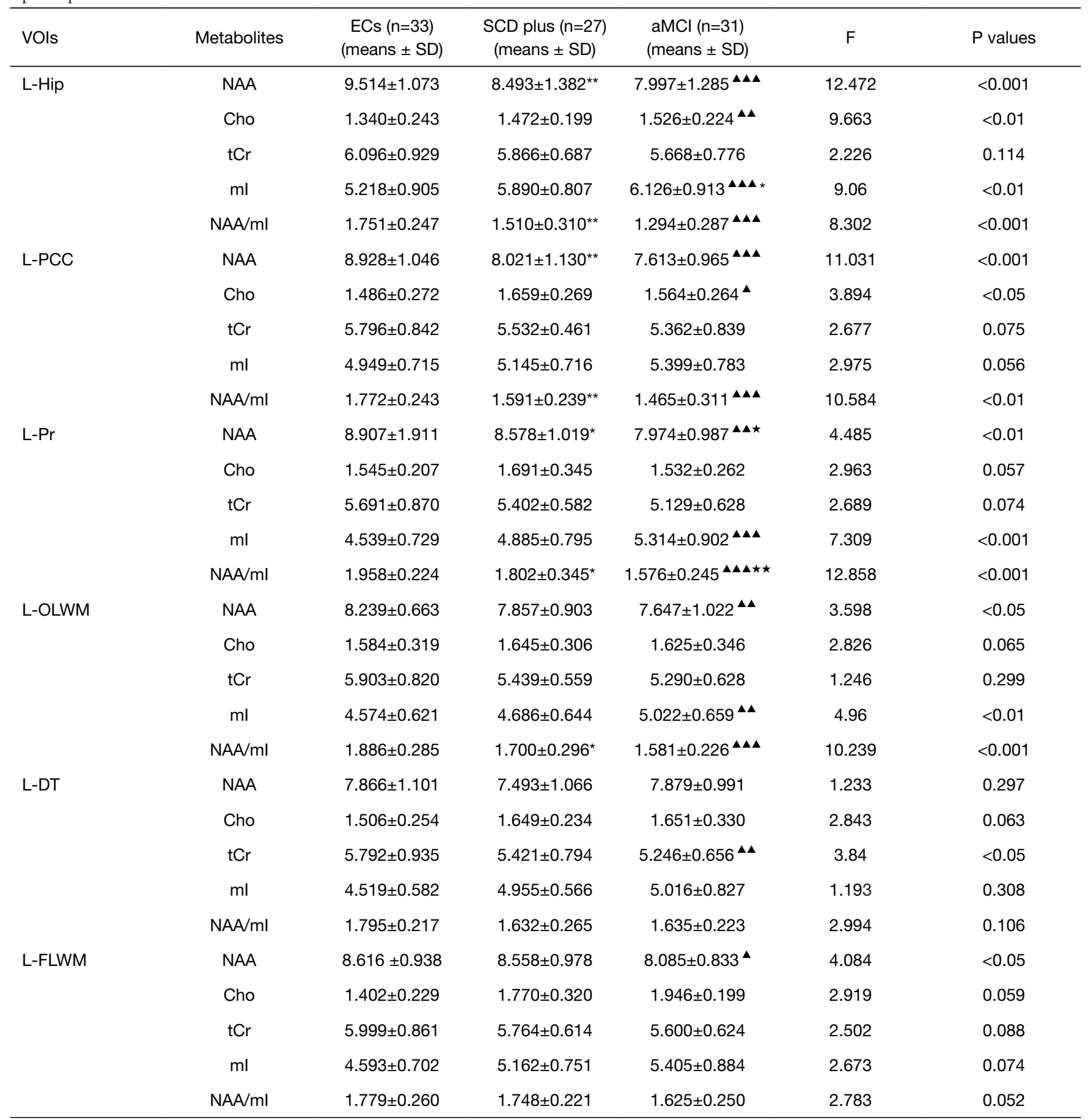

Table 2 (continued) 
Table 2 (continued)

\begin{tabular}{|c|c|c|c|c|c|c|}
\hline VOls & Metabolites & $\begin{array}{c}\text { ECs }(n=33) \\
(\text { means } \pm S D)\end{array}$ & $\begin{array}{c}\text { SCD plus }(n=27) \\
\text { (means } \pm S D)\end{array}$ & $\begin{array}{c}\text { aMCl }(n=31) \\
\text { (means } \pm \text { SD) }\end{array}$ & $\mathrm{F}$ & $P$ values \\
\hline \multirow[t]{2}{*}{ L-LN } & NAA & $8.245 \pm 1.056$ & $7.739 \pm 0.982$ & $7.634 \pm 1.060^{\Delta}$ & 3.172 & $<0.05$ \\
\hline & $\mathrm{tCr}$ & $6.110 \pm 0.888$ & $5.725 \pm 0.672$ & $5.682 \pm 0.672$ & 3.077 & 0.051 \\
\hline \multirow[t]{3}{*}{ L-CNC } & NAA & $8.761 \pm 1.131$ & $8.172 \pm 0.824$ & $7.910 \pm 1.181^{\wedge}$ & 3.327 & $<0.05$ \\
\hline & Cho & $1.516 \pm 0.212$ & $1.707 \pm 0.331$ & $1.579 \pm 0.297$ & 1.462 & 0.271 \\
\hline & $\mathrm{tCr}$ & $5.813 \pm 0.779$ & $5.448 \pm 0.484$ & $5.586 \pm 0.725$ & 2.184 & 0.119 \\
\hline
\end{tabular}

ECs, elderly controls; SCD plus, subjective cognitive decline plus; aMCl, amnestic mild cognitive impairment; L-Hip, left hippocampus; L-PCC, left posterior cingulate cortex; L-Pr, left precuneus; L-OLWM, left white matter of occipital lobe; L-DT, left dorsal thalamus; L-FLWM, left white matter of frontal lobe; L-LN, left lenticular nucleus; L-CNC, left caput nuclei caudati. Values are presented as mean \pm SD. NAA, N-acetylaspartate; tCr, total creatine; Cho, choline; ml, myoinositol. SCD plus vs. ECs: ${ }^{*} \mathrm{P}<0.05,{ }^{\star} \mathrm{P}<0.01 ; \mathrm{aMCl}$ vs. ECs: ${ }^{\boldsymbol{}} \mathrm{P}<0.05$, ${ }^{\Delta \Delta} \mathrm{P}<0.01,{ }^{\Delta \Delta \Delta} \mathrm{P}<0.001$; aMCl vs. SCD plus: ${ }^{\star} \mathrm{P}<0.05,{ }^{\star \star} \mathrm{P}<0.01$.

Table 3 Group differences in metabolite concentrations (mmol/L) and NAA/mI ratio in left volume of interests (VOIs) of ECs, SCD plus and aMCI participants

\begin{tabular}{|c|c|c|c|c|c|c|c|}
\hline VOls & Metabolites & \multicolumn{2}{|l|}{ SCD plus vs. ECs } & \multicolumn{2}{|l|}{ aMCI vs. ECs } & \multicolumn{2}{|l|}{ aMCI vs. SCD plus } \\
\hline \multirow[t]{4}{*}{ L-Hip } & NAA & $-1.012(-1.656,-0.387)$ & $<0.01$ & $-1.517(-2.108,-0.926)$ & $<0.001$ & $-0.496(-1.200,0.208)$ & 0.164 \\
\hline & Cho & $0.131(-0.017,0.246)$ & 0.073 & $0.185(0.027,0.345)$ & $<0.01$ & $0.054(-0.098,0.206)$ & 0.698 \\
\hline & $\mathrm{tCr}$ & - & & - & - & - & - \\
\hline & $\mathrm{NAA} / \mathrm{ml}$ & $-0.241(-0.389,-0.094)$ & $<0.01$ & $-0.257(-0.393,-0.121)$ & $<0.001$ & $-0.016(-0.175,0.145)$ & 0.845 \\
\hline \multirow[t]{3}{*}{ L-PCC } & NAA & $-0.679(-1.241,-0.117)$ & $<0.01$ & $-1.517(-2.108,-0.926)$ & $<0.001$ & $-0.534(-0.109,0.015)$ & 0.056 \\
\hline & Cho & $0.061(-0.101,0.223)$ & 0.453 & $0.221(0.054,0.388)$ & $<0.05$ & $0.160(-0.012,0.331)$ & 0.067 \\
\hline & $\mathrm{tCr}$ & - & - & - & - & - & - \\
\hline \multirow[t]{5}{*}{ L-Pr } & NAA & $-0.329(-0.835,-0.177)$ & $<0.05$ & $-0.933(-1.409,-0.457)$ & $<0.01$ & $-0.604(-1.134,-0.074)$ & $<0.05$ \\
\hline & Cho & - & - & - & - & - & - \\
\hline & $\mathrm{tCr}$ & - & - & - & - & - & - \\
\hline & $\mathrm{ml}$ & $0.346(-0.053,0.745)$ & 0.086 & $0.274(0.186,0.363)$ & $<0.001$ & $0.428(-0.018,0.875)$ & 0.059 \\
\hline & $\mathrm{NAA} / \mathrm{ml}$ & $-0.157(-0.312,-0.001)$ & $<0.05$ & $-0.382(-0.449,-0.265)$ & $<0.001$ & $-0.226(-0.386,-0.065)$ & $<0.01$ \\
\hline
\end{tabular}

Table 3 (continued) 
Table 3 (continued)

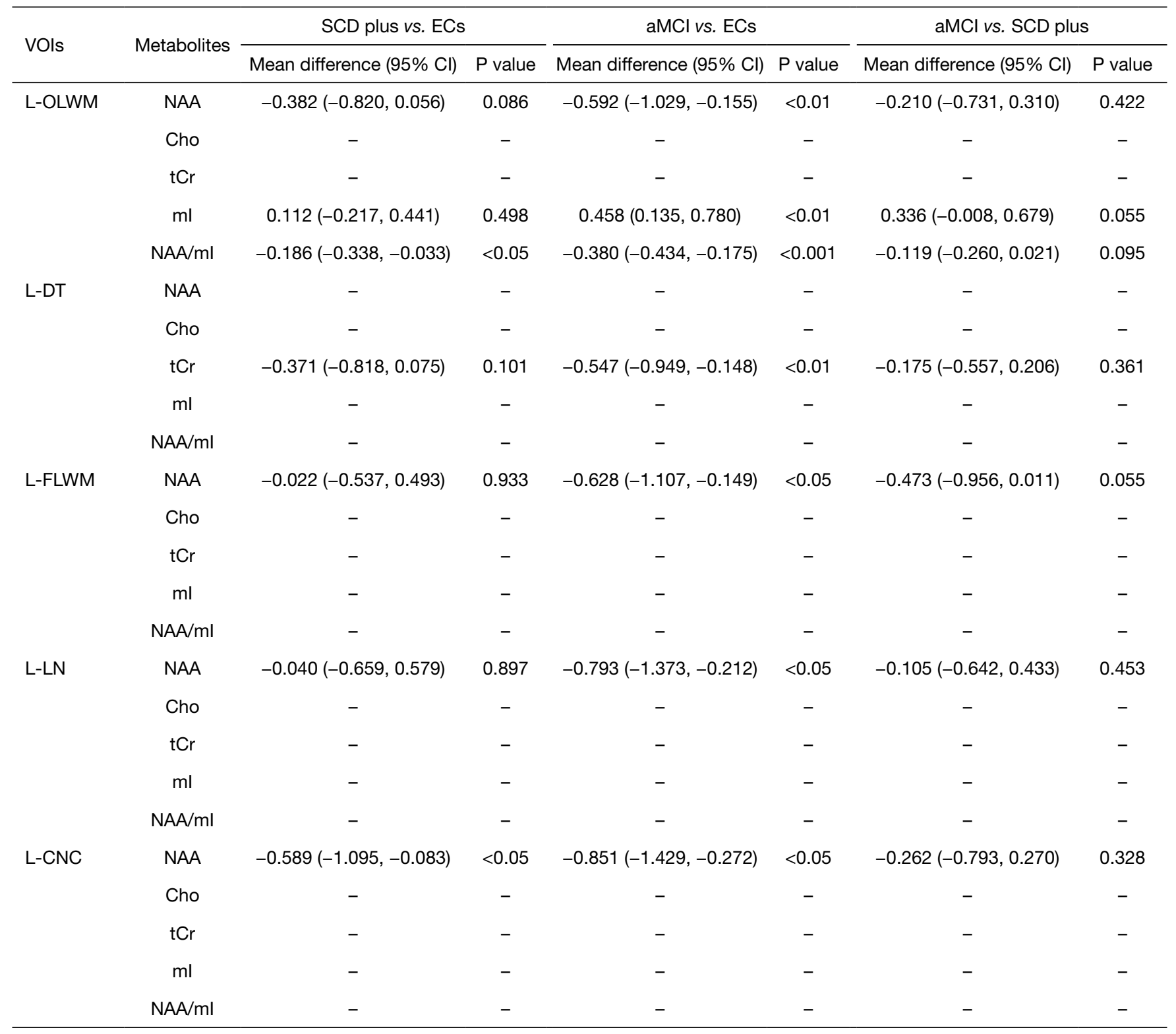

ECs, elderly controls; SCD plus, subjective cognitive decline plus; aMCl, amnestic mild cognitive impairment; Cl, confidence interval; L-Hip, left hippocampus; L-PCC, left posterior cingulate cortex; L-Pr, left precuneus; L-OLWM, left white matter of occipital lobe; L-DT, left dorsal thalamus; L-FLWM, left white matter of frontal lobe; L-LN, left lenticular nucleus; L-CNC, left caput nuclei caudate; NAA, N-acetylaspartate; $\mathrm{tCr}$, total creatine; Cho, choline; $\mathrm{ml}$, myoinositol. 
Table 4 Concentrations of metabolites (unit, $\mathrm{mmol} / \mathrm{L}$ ) and $\mathrm{NAA} / \mathrm{mI}$ ratios derived from the right volume of interests (VOIs) of ECs, SCD plus and aMCI participants

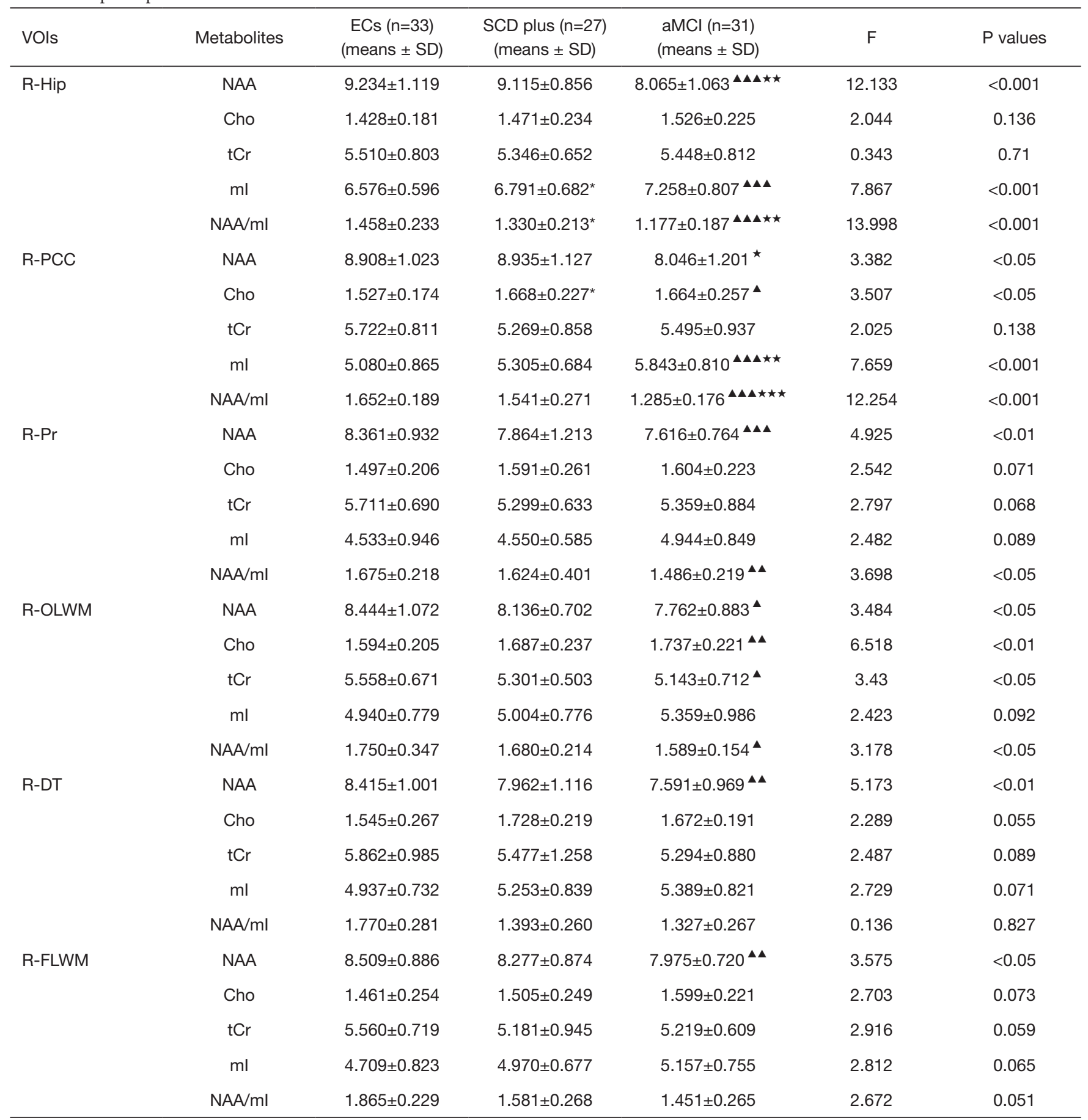

Table 4 (continued) 
Table 4 (continued)

\begin{tabular}{|c|c|c|c|c|c|c|}
\hline VOls & Metabolites & $\begin{array}{c}\text { ECs }(n=33) \\
\text { (means } \pm S D)\end{array}$ & $\begin{array}{l}\text { SCD plus }(n=27) \\
\text { (means } \pm \text { SD) }\end{array}$ & $\begin{array}{c}\text { aMCl }(n=31) \\
(\text { means } \pm \text { SD) }\end{array}$ & $\mathrm{F}$ & $P$ values \\
\hline \multirow[t]{3}{*}{ R-LN } & NAA & $8.245 \pm 1.056$ & $7.739 \pm 0.982$ & $7.634 \pm 1.060$ & 2.272 & 0.061 \\
\hline & $\mathrm{tCr}$ & $6.260 \pm 1.178$ & $5.764 \pm 0.726$ & $5.692 \pm 1.300$ & 2.465 & 0.091 \\
\hline & $\mathrm{ml}$ & $4.727 \pm 1.246$ & $5.367 \pm 1.053$ & $5.186 \pm 1.017$ & 2.39 & 0.074 \\
\hline \multirow[t]{3}{*}{$\mathrm{R}-\mathrm{CNC}$} & NAA & $8.354 \pm 0.945$ & $8.023 \pm 1.096$ & $7.995 \pm 0.853^{\wedge}$ & 4.253 & $<0.05$ \\
\hline & Cho & $1.590 \pm 0.175$ & $1.697 \pm 0.247$ & $1.701 \pm 0.202$ & 2.025 & 0.056 \\
\hline & $\mathrm{tCr}$ & $5.801 \pm 1.055$ & $5.541 \pm 0.867$ & $5.411 \pm 0.645$ & 2.925 & 0.199 \\
\hline
\end{tabular}

ECs, elderly controls; SCD plus, subjective cognitive decline plus; aMCl, amnestic mild cognitive impairment; R-Hip, right hippocampus; $\mathrm{R}-\mathrm{PCC}$, right posterior cingulate cortex; R-Pr, right precuneus; R-OLWM, right white matter of occipital lobe; R-DT, right dorsal thalamus; R-FLWM, right white matter of frontal lobe; R-LN, right lenticular nucleus; R-CNC, right caput nuclei caudati. Values are presented as mean $\pm \mathrm{SD}$. NAA, $\mathrm{N}$-acetylaspartate; $\mathrm{tCr}$, total creatine; Cho, choline; ml, myoinositol. SCD plus vs. ECs: "P<0.05, " $\mathrm{P}<0.01$; aMCl vs. ECs: ${ }^{\boldsymbol{\Delta}} \mathrm{P}<0.05,{ }^{\boldsymbol{\Delta}} \mathrm{P}<0.01,{ }^{\boldsymbol{\Delta} \Delta \mathbf{\Delta}} \mathrm{P}<0.001$; aMCl vs. SCD plus: ${ }^{\star} \mathrm{P}<0.05,{ }^{\star \star} \mathrm{P}<0.01,{ }^{\star \star \star} \mathrm{P}<0.001$.

Table 5 Group differences in metabolite concentration ( $\mathrm{mmol} / \mathrm{L}$ ) and $\mathrm{NAA} / \mathrm{mI}$ ratio in right volume of interests (VOIs) of ECs, SCD plus and aMCI participants

\begin{tabular}{|c|c|c|c|c|c|c|c|}
\hline VOls & Metabolites & \multicolumn{2}{|l|}{ SCD plus vs. ECs } & \multicolumn{2}{|l|}{ aMCI vs. ECs } & \multicolumn{2}{|l|}{ aMCl vs. SCD plus } \\
\hline \multirow[t]{4}{*}{ R-Hip } & NAA & $-0.119(-0.630,0.392)$ & 0.646 & $-1.169(-1.714,-0.624)$ & $<0.001$ & $-1.050(-1.556,-0.545)$ & $<0.01$ \\
\hline & Cho & - & - & - & - & - & - \\
\hline & $\mathrm{tCr}$ & - & - & - & - & - & - \\
\hline & $\mathrm{NAA} / \mathrm{ml}$ & $-0.128(-0.243,-0.012)$ & $<0.05$ & $-0.281(-0.386,-0.176)$ & $<0.001$ & $-0.153(-0.259,-0.047)$ & $<0.01$ \\
\hline \multirow[t]{3}{*}{ R-PCC } & NAA & $-0.050(-0.560,0.660)$ & 0.985 & $-0.739(-1.469,0.009)$ & 0.053 & $-0.739(-1.394,-0.833)$ & $<0.05$ \\
\hline & Cho & $0.159(0.052,0.266)$ & $<0.05$ & $0.127(0.017,0.238)$ & $<0.05$ & $0.032(-0.096,0.159)$ & 0.622 \\
\hline & $\mathrm{tCr}$ & - & - & - & - & - & - \\
\hline \multirow[t]{5}{*}{$\mathrm{R}-\mathrm{Pr}$} & NAA & $-0.309(-0.770,0.153)$ & 0.186 & $-0.582(-1.177,-0.192)$ & $<0.01$ & $-0.373(-0.790,0.044)$ & 0.087 \\
\hline & Cho & - & - & - & - & - & - \\
\hline & $\mathrm{tCr}$ & - & - & - & - & - & - \\
\hline & $\mathrm{ml}$ & - & - & - & - & - & - \\
\hline & $\mathrm{NAA} / \mathrm{ml}$ & $-0.051(-0.225,0.124)$ & 0.56 & $-0.189(-0.298,-0.079)$ & $<0.01$ & $-0.138(-0.314,0.087)$ & 0.119 \\
\hline
\end{tabular}

Table 5 (continued) 
Table 5 (continued)

\begin{tabular}{|c|c|c|c|c|c|c|c|}
\hline VOls & Metabolites & \multicolumn{2}{|l|}{ SCD plus vs. ECs } & \multicolumn{2}{|l|}{ aMCl vs. ECs } & \multicolumn{2}{|l|}{ aMCI vs. SCD plus } \\
\hline \multirow[t]{4}{*}{ R-OLWM } & NAA & $-0.174(-0.671,0.323)$ & 0.486 & $-0.474(-0.864,-0.083)$ & $<0.05$ & $-0.494(-0.937,-0.051)$ & 0.079 \\
\hline & Cho & $0.094(-0.023,0.210)$ & 0.112 & $0.143(0.363,0.250)$ & $<0.01$ & $0.049(-0.072,0.171)$ & 0.418 \\
\hline & $\mathrm{tCr}$ & $-0.257(-0.561,0.046)$ & 0.095 & $-0.416(-0.761,-0.069)$ & $<0.05$ & $-0.158(-0.480,0.163)$ & 0.328 \\
\hline & $\mathrm{NAA} / \mathrm{ml}$ & $-0.070(-0.217,0.077)$ & 0.344 & $-0.161(-0.295,-0.027)$ & $<0.05$ & $-0.091(-0.191,0.008)$ & 0.072 \\
\hline \multirow[t]{3}{*}{ R-DT } & NAA & $-0.452(-1.007,0.102)$ & 0.108 & $-0.823(-1.315,-0.331)$ & $<0.01$ & $-0.371(-0.926,0.184)$ & 0.185 \\
\hline & Cho & - & - & - & - & - & - \\
\hline & $\mathrm{tCr}$ & - & - & - & - & - & - \\
\hline \multirow[t]{5}{*}{ R-FLWM } & NAA & $-0.232(-0.667,0.203)$ & 0.289 & $-0.534(-0.914,-0.155)$ & $<0.01$ & $-0.302(-0.731,0.127)$ & 0.163 \\
\hline & Cho & - & - & - & - & - & - \\
\hline & $\mathrm{tCr}$ & - & - & - & - & - & - \\
\hline & $\mathrm{ml}$ & - & - & - & - & - & - \\
\hline & $\mathrm{NAA} / \mathrm{ml}$ & - & - & - & - & - & - \\
\hline \multirow[t]{3}{*}{ R-LN } & NAA & - & - & - & - & - & - \\
\hline & Cho & - & - & - & - & - & - \\
\hline & $\mathrm{tCr}$ & - & - & - & - & - & - \\
\hline \multirow{2}{*}{$\mathrm{R}-\mathrm{CNC}$} & $\mathrm{ml}$ & - & - & - & - & - & - \\
\hline & $\mathrm{NAA} / \mathrm{ml}$ & - & - & - & - & - & - \\
\hline
\end{tabular}

ECs, elderly controls; SCD plus, subjective cognitive decline plus; aMCl, amnestic mild cognitive impairment; Cl, confidence interval; R-Hip, right hippocampus; R-PCC, right posterior cingulate cortex; R-Pr, right precuneus; R-OLWM, right white matter of occipital lobe; R-DT, right dorsal thalamus; R-FLWM, right white matter of frontal lobe; R-LN, right lenticular nucleus; R-CNC, right caput nuclei caudate; NAA, $\mathrm{N}$-acetylaspartate; $\mathrm{tCr}$, total creatine; Cho, choline; $\mathrm{ml}$, myoinositol. 


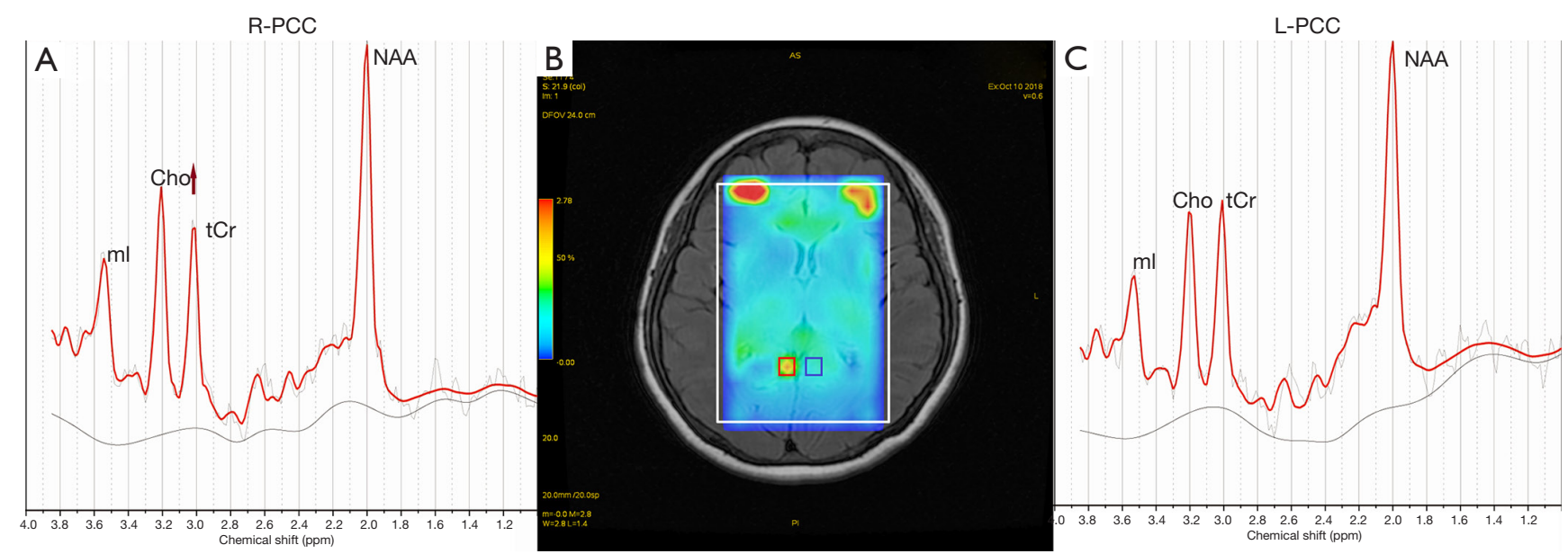

Figure 3 Examples of the Multi-voxel 1H-MRS spectra of the right and left PCC from SCD plus participant (A and C) and the axial metabolic maps of choline (B). Cho increased significantly in the right PCC, while no significant difference in the left PCC. L-PCC, left posterior cingulate cortex; R-PCC, right posterior cingulate cortex; Cho, choline; NAA, N-acetylaspartate; tCr, total creatine; mI, myoinositol.

decreased in the left Hip, left PCC, left Pr, left OLWM, and right Hip. The concentration of Cho was observed to only increase in the right PCC. Figure 3 shows an example images of increased Cho levels in the right PCC in patients with SCD plus, while no significant difference was observed in the left PCC.

Compared with ECs group, aMCI group showed obvious lower NAA values in the bilateral Hip, bilateral Pr, bilateral OLWM, bilateral CNC, left PCC, left LN, left FLWM, and right DT. Cho levels were observed increased in the bilateral PCC, left Hip, and right OLWM. The mI levels were found increased in the bilateral Hip, left Pr, left OLWM, and right PCC. A decrease in $\mathrm{tCr}$ values was observed in the left DT and right OLWM. The metabolite ratios of $\mathrm{NAA} / \mathrm{mI}$ were observed to decline in the bilateral Hip, bilateral PCC, bilateral Pr, and bilateral OLWM.

Compared with SCD plus group, aMCI group had decreased concentrations of NAA in the left Pr, right Hip, and right PCC. Increased $\mathrm{mI}$ concentrations were found in the left Hip and the right PCC. The NAA/mI ratio was decreased in the left Pr, right Hip, and right PCC. No significant metabolic differences in Cho and $\mathrm{tCr}$ were observed in any VOIs between the two groups.

Heat maps were constructed to visualize the distinction power of neurometabolites of interest (NAA, mI, and $\mathrm{NAA} / \mathrm{mI})$ among the three groups, as shown in Figure $4 A, B, C$.

\section{Predictive accuracy using combinations of metabolites differences and correlation analyses for data from patients with SCD plus}

The NAA/mI ratio mentioned in previous literature was considered a screening marker for predicting $\mathrm{AD}$ using neuroimaging (45-47). Our results showed that the differences in brain neurometabolites in patients with SCD plus and aMCI are multi-regional, especially in areas of atrophy associated with $\mathrm{AD}$-sensitive brain located on the hippocampus, posterior cingulate, precuneus, with a left-sided predominance (48). In addition to the differences between groups with statistically significant $(\mathrm{P}<0.05), \mathrm{P}$ values of AUC were also set as less than 0.05 in differentiating SCD plus from ECs or aMCI groups. Only when the thresholds passed, the corresponding metabolites were selected for ROC analysis. Our results showed that the $\mathrm{P}$ values of the AUC values on NAA [left Hip, $\mathrm{P}<0.01$; left $\mathrm{PCC}, \mathrm{P}<0.01$; left $\mathrm{Pr}, \mathrm{P}<0.05$; left $\mathrm{CNC}, \mathrm{P}<0.05$; ] and $\mathrm{mI}$ value (right Hip, $\mathrm{P}<0.05$ ) were statistically significant in distinguishing SCD plus from ECs. Although the $\mathrm{P}$ value of Cho level in the right PCC showed statistical differences between SCD plus and ECs groups $(\mathrm{P}<0.05)$, the $\mathrm{P}$ value of the AUC is 0.053 ( $\mathrm{P}>0.05)$; hence, this index was omitted. As to other indices, the above conditions were all met between the SCD plus and ECs/aMCI; hence, the ROC analysis could be performed. Thus, we sought to investigate whether 




B

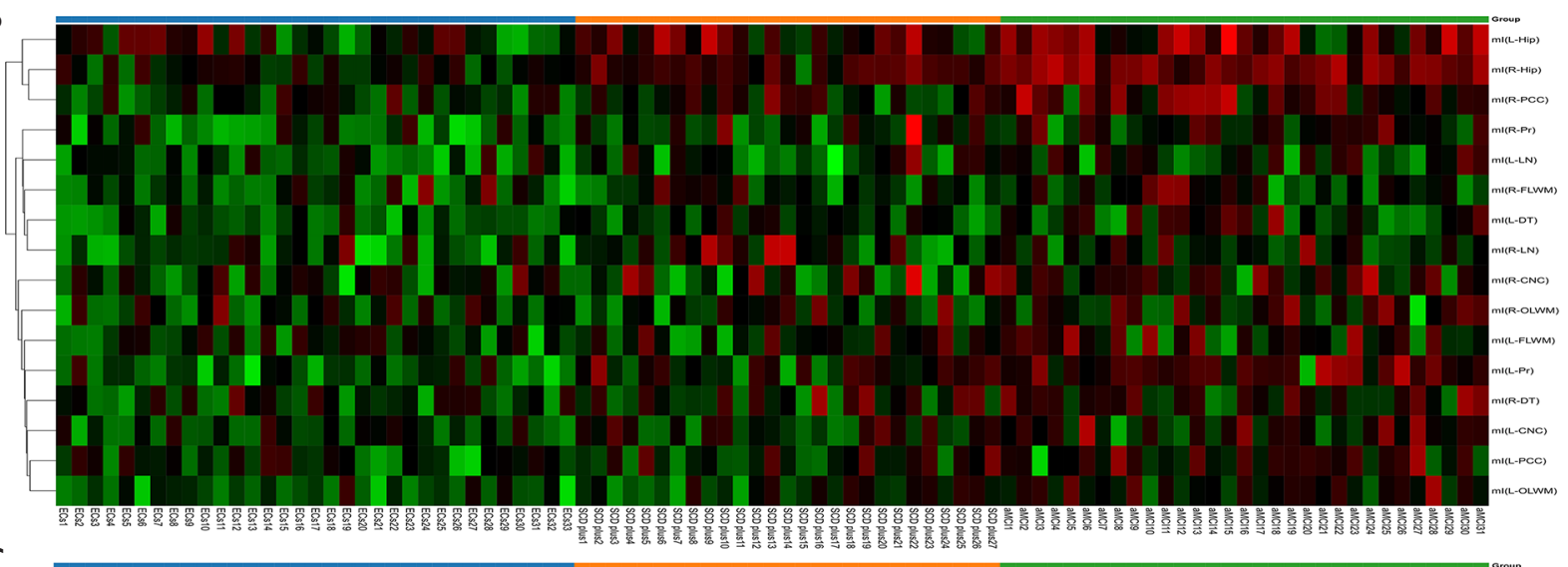

C

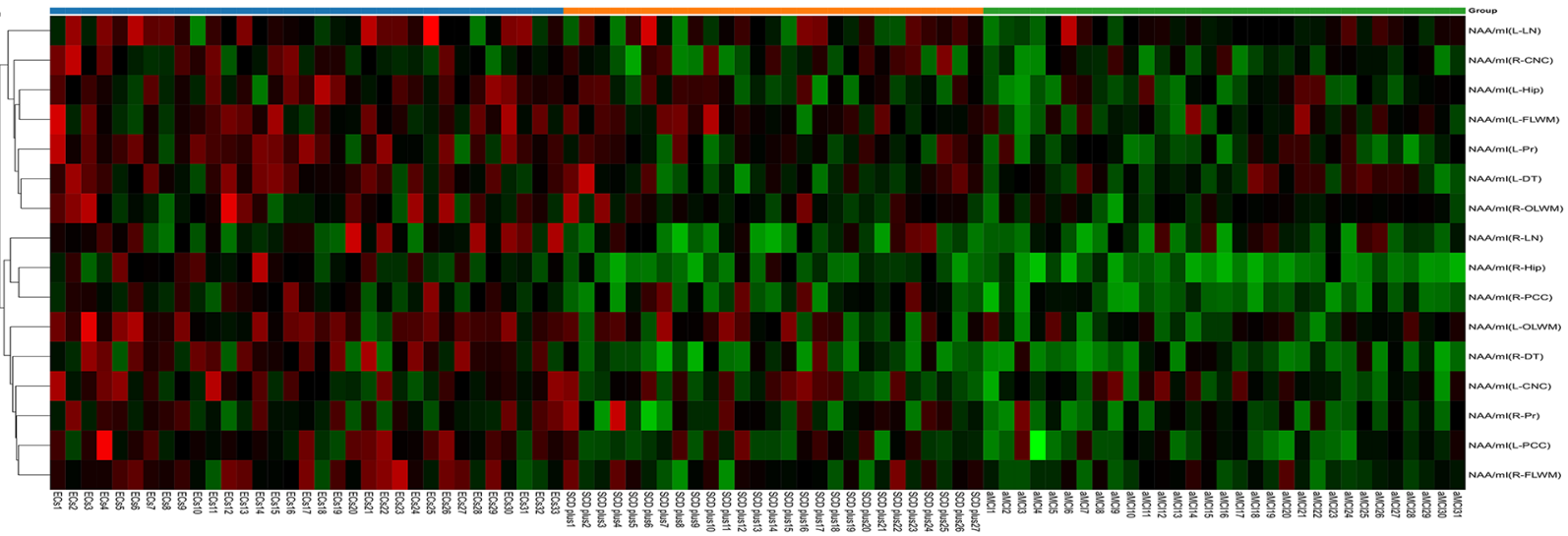

Figure 4 Heat maps to visualize the distinction power of N-acetylaspartate (NAA) (A), myoinositol (mI) (B) and NAA/mI (C) among the three groups. Each column represents one volume of interest (VOI), and each row represents one sample. The intensity increases from green (relatively decreased) to red (relatively increased). In the top bar, the blue color indicates elderly controls (ECs), the pink color indicates subjective cognitive decline plus (SCD plus) group, and the green color indicates amnestic mild cognitive impairment (aMCI) group.

the differentiation SCD plus from ECs or aMCI groups may be estimated more accurately using the concentration of metabolites of NAA combined with $\mathrm{mI}$ or the NAA $/ \mathrm{mI}$ ratio. Supplementary file 3 showed the basic process flow of the RF algorithm. Figure S3A,B and Figure S3C,D showed the AUC area and importance measurements from the RF based on the testing dataset in discriminating participants with SCD plus from ECs using NAA and $\mathrm{mI}$ values and $\mathrm{NAA} / \mathrm{mI}$ ratio, respectively. Figure S4A,B and Figure S4C,D showed the AUC area and importance measurements from 
A

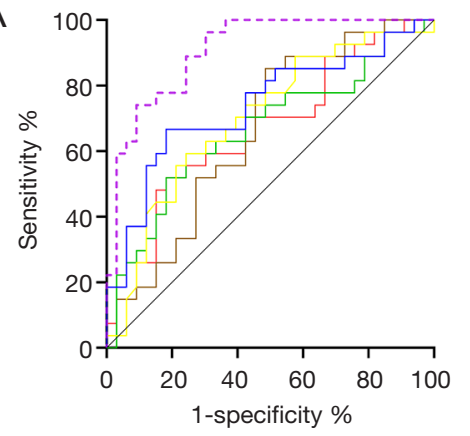

B

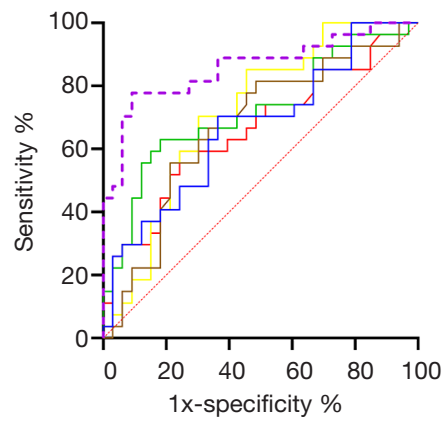

- - Combination AUC $=0.895$ (95\% Cl: 0.817-0.973)

— L-Hip (NAA) AUC $=0.712$ (95\% Cl: 0.620-0.803)

L-PCC (NAA) AUC $=0.700$ (95\% Cl: 0.565-0.834)

— L-Pr (NAA) AUC $=0.672(95 \% \mathrm{Cl}: 0.530-0.813)$

- L-CNC (NAA) AUC $=0.663$ (95\% Cl: $0.526-0.800$ )

— R-Hip (ml) AUC $=0.666$ (95\% Cl: $0.525-0.807)$

- - Combination AUC $=0.860$ (95\% Cl: 0.759-0.960)

— L-Hip (NAA/ml) AUC $=0.668$ (95\% Cl: 0.529-0.806)

— R-Hip (NAA/ml) AUC $=0.718$ (95\% Cl: 0.584-0.853)

— L-PCC (NAA/ml) AUC $=0.686$ (95\% Cl: 0.549-0.823) L-Pr (NAA/ml) AUC $=0.663$ (95\% Cl: 0.522-0.804)

— L-OLWM (NAA/ml) AUC $=0.671$ (95\% Cl: 0.531-0.811)

Figure 5 ROC of the discriminatory power of the combined marker panel from SCD plus to ECs. The combined area under curve (AUC) values in differentiating participants with SCD plus from ECs acquired by NAA and mI levels (A: AUC =0.895). The combined AUC values in differentiating participants with SCD plus from ECs acquired by NAA/mI ratios (B: AUC =0.860). The horizontal and vertical coordinates represent specificity and sensitivity, respectively. ECs, elderly controls; SCD plus, subjective cognitive decline plus; R-Hip, right hippocampus; L-Hip, left hippocampus; L-PCC, left posterior cingulate cortex; L-Pr, left precuneus; L-OLWM, left white matter of occipital lobe; L-CNC, left caput nuclei caudati; NAA, N-acetylaspartate; mI, myoinositol.

the RF based on the testing dataset in discriminating SCD plus from aMCI using NAA and $\mathrm{mI}$ values and $\mathrm{NAA} / \mathrm{mI}$ ratio, respectively. The results of testing dataset showed that the AUC values for final models were not overfitting. Therefore, the AUC values based on significant group differences could be used for subsequent statistical analysis. The combined AUC levels in distinguishing SCD plus from ECs participants acquired by NAA values in the left Hip, left $\mathrm{Pr}$, left PCC, left $\mathrm{CNC}$ and the $\mathrm{mI}$ value in the right Hip increased to 0.895 [Figure $5 A$, 95\% confidence interval (CI): 0.817-0.973], whereas the combined AUC levels in differentiating SCD plus from ECs participants acquired by the $\mathrm{NAA} / \mathrm{mI}$ ratios in the left Hip, left Pr, left PCC, left OLWM and the right Hip added to 0.860 (Figure $5 B$, 95\% CI: 0.759-0.960). Likewise, the combined AUC levels in differentiating participants with SCD plus from aMCI acquired by the NAA levels in the left Pr, right Hip, right PCC, together with $\mathrm{mI}$ values in the left Hip and right PCC added up to 0.892 (Figure $6 A, 95 \%$ CI: 0.811-0.973). The combined AUC values in distinguishing SCD plus from aMCI participants acquired by the NAA $/ \mathrm{mI}$ ratios in the left Pr, right Hip and right PCC added up to 0.836 (Figure $6 B, 95 \%$ CI: $0.735-0.938$ ). These results revealed that the AUC values obtained by combining the levels of NAA with $\mathrm{mI}$ made more significant contributions to the classification than those calculated using the $\mathrm{NAA} / \mathrm{mI}$ ratios. Besides, correlation tests between the key neuropsychological scores and ${ }^{1} \mathrm{H}$-MRS metabolites were conducted for SCD plus group. Positive correlations were found between AVLT-De scores and NAA levels in the left Hip (Figure $7 A, \mathrm{r}=0.386$, $\mathrm{P}=0.047$ ) and in the left PCC (Figure $7 B, \mathrm{r}=0.395, \mathrm{P}=0.042$ ). While AVLT-De scores were only positively associated with $\mathrm{NAA} / \mathrm{mI}$ ratio in the left Hip (Figure $7 C, \mathrm{r}=0.416, \mathrm{P}=0.031$ ) of SCD plus group. There were no significant correlations $(\mathrm{P}>0.05)$ between AVLT-De scores and the rest of MRS metrics in SCD plus participants. 

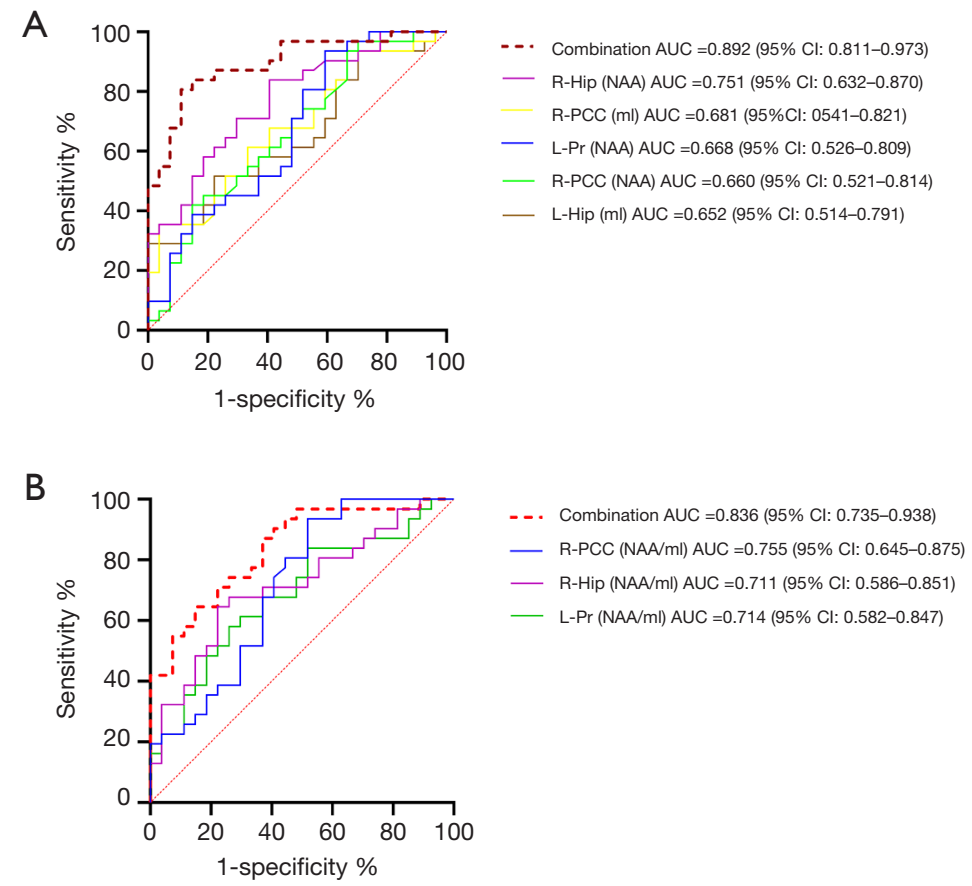

Figure 6 ROC of the discriminatory power of the combined marker panel from SCD plus to aMCI. The combined area under curve (AUC) values in differentiating participants with SCD plus from aMCI acquired by NAA with mI values (A: AUC =0.892). The combined AUC values in differentiating participants with SCD plus from aMCI acquired by NAA/mI ratios (B: AUC =0.836). The horizontal and vertical coordinates represent specificity and sensitivity, respectively. aMCI, amnestic mild cognitive impairment; SCD plus, subjective cognitive decline plus; R-Hip, right hippocampus; R-PCC, right posterior cingulate cortex; L-Pr, left precuneus; NAA, N-acetylaspartate; mI, myoinositol.
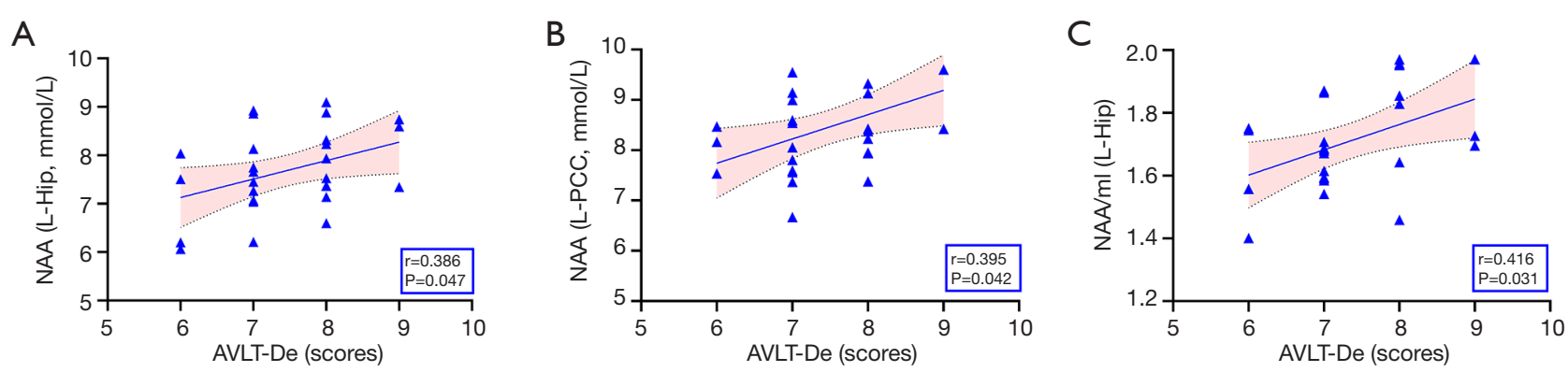

Figure 7 Correlations between imaging metrics and AVLT delayed recall scores for SCD plus. The horizontal coordinate represents AVLTDe scores and the vertical coordinates represent metabolite concentration or metabolite ratio. AVLT-De scores positively correlated with NAA levels (mmol/L) in L-Hip (A) and L-PCC (B). AVLT-De scores positively correlated with NAA/mI ratio in L-Hip (C). SCD plus, subjective cognitive decline plus; L-Hip, left hippocampus; L-PCC, left posterior cingulate cortex; NAA, N-acetylaspartate; mI, myoinositol; AVLT-De, Auditory Verbal Learning Test delayed recall. 


\section{Discussion}

In our study, ${ }^{1} \mathrm{H}-\mathrm{SVS}$ and ${ }^{1} \mathrm{H}-\mathrm{MVS}$ were employed to generate and output plenty of rich metabolite data by combined with many different brain regional distributions' characteristics of MRS. This allowed us to explore the changes in metabolic distribution in different brain areas and acquire more accurate bioinformation for discrimination and diagnosis using this practical technique. Firstly, our main results suggested that patients with SCD plus reflect brain neurometabolic changes in many brain areas, especially in the Hip and PCC. Patients with aMCI revealed more obvious difference compared to individuals with SCD plus and ECs in many brain areas. These findings also suggested that SCD plus participants could share a similar tendency of brain neurometabolic differences with individuals with aMCI. Compared with ECs, Cho was observed to only increase in the right PCC in SCD plus, while $\mathrm{tCr}$ values were observed to decrease in the left DT and right OLWM in aMCI. Secondly, NAA concentrations may be used as one of the earliest potential neuroimaging markers at this stage, while $\mathrm{NAA} / \mathrm{mI}$ ratio could be more suitable for monitoring AD progression. Moreover, the AVLT-De scores were positively associated with the NAA levels in the left Hip and left PCC in SCD plus group, whereas only the NAA/mI ratio was positively associated with the AVLT-De scores in the left Hip.

In our previous studies and other studies, patients with aMCI/MCI had been reported to have different metabolite distribution and aberrant metabolite patterns in multiple regions of the brain $(30,34,49)$. In this study, we observed a different metabolite distribution, such as in the Hip, Pr, PCC, OLWM, DT, LN, CNC, and FLWM in aMCI participants, reflecting metabolic differences in these regions. Our results are consistent with previous research and extend the reports on the metabolic differences to include regions other than PCC and Hip in the likelihood of $\mathrm{AD}$. However, our main purpose was to explore the differences in the levels of several brain metabolites in patients with SCD plus. Additionally, we also compared the advantages of absolute quantification of metabolites (NAA and $\mathrm{mI}$ ) and metabolite ratios $(\mathrm{NAA} / \mathrm{mI})$ in distinguishing SCD plus group from aMCI or ECs individuals.

NAA is abundant in the human brain and metabolized in the mitochondria of neurons from aspartic acid and acetylcoenzyme A. NAA is considered a part of the neuronal mitochondrial energetic metabolism through Kreb's cycle. The Kreb's cycle guarantees an additional supply of energy by the oxidation of glutathione via aspartate amino transferase that leads to produced alpha-ketoglutarate and NAA (50). The energy metabolism decline, which is known to take place early in the process of $\mathrm{AD}$ may also depress the NAA concentration (51). Therefore, NAA decline is closely related to the ATP synthesis in mitochondrial dysfunction. Since SCD plus may lead to a higher risk of a very early stage of $\mathrm{AD}$, minor alterations in the NAA levels detected could be the early response to brain energy depletion. Detection of the alterations in NAA seem to be an effective way in evaluation of individuals at high-risk for dementia since it might reflect an early neuroimaging marker of energy metabolism injury in the brain, a factor referred to the pathological mechanism of $\mathrm{AD}(52,53)$.

In this study, elevated mI was only found in the left Hip in participants with SCD plus compared to the ECs. The $\mathrm{mI}$ concentration changes were observed in two brain regions (right Hip and right PCC) between SCD plus and aMCI participants. Further, our study showed that increased $\mathrm{mI}$ values were extended to three regions (bilateral Hip and right PCC) in participants with aMCI compared to those in ECs. Increased $\mathrm{mI}$ may indicate glial activation or proliferation. In the neuropathological process of $\mathrm{AD}$, gliosis was associated with neuronal loss, which may result in disruption of the osmotic balance (18). $\mathrm{mI}$ might accumulate to regulate the osmotic balance to maintain cell volume homeostasis in the neuroglial cells (54). We speculate that the combined effect of gliosis and osmotic stress may be responsible for the elevated $\mathrm{mI}$ levels observed in the left Hip region. A study reported that the $\mathrm{mI}$ values were already increased during the preclinical stages of $\mathrm{AD}$, especially in APOE $\varepsilon 4$ carriers, indicating that $\mathrm{mI}$ levels may reveal brain region consequences of APOE $\varepsilon 4$ before detectable amyloid-related pathology (55). Compared with the concentration of NAA, the brain regions involved with changes in $\mathrm{mI}$ are few in patients with SCD plus. A recent longitudinal study (18) and a seven-year follow-up study (47) have shown that $\mathrm{mI}$ and $\mathrm{NAA} / \mathrm{mI}$ ratio may be useful in predicting $\mathrm{AD}$ progression. In this study, we found $\mathrm{mI}$ values gradually increased and the number of brain regions with elevated $\mathrm{mI}$ levels increased from ECs to aMCI. Our results indicated that alterations of NAA were detected prior to those in $\mathrm{mI}$. One study has shown that decreased NAA and increased $\mathrm{mI}$ represents lack of correlation and reflected different pathologic $\mathrm{AD}$ processes (46). Our study also showed that NAA and $\mathrm{mI}$ levels may represent different processes underlying different stages of $\mathrm{AD}$. Hence, NAA could be served as an early diagnosis marker, while mI 
could be better suited for monitoring AD progression. However, long-term follow-up studies are also needed to determine whether $\mathrm{NAA} / \mathrm{mI}$ is suitable for monitoring $\mathrm{AD}$ progression.

As precursors of the neurotransmitter acetylcholine, elevated Cho values may be associated with the increase of cell membrane phospholipid turnover (56). In this study, increased Cho value was only observed in the right PCC in SCD plus compared with ECs individuals. The PCC is a limbic cortical region that experiences neuronal density loss and mitochondrial energy metabolism decline as well as cognitive dysfunction in $\mathrm{AD}$ (57). Therefore, the increased Cho value in the PCC of patients with SCD plus may reflect a compensatory mechanism of decreased choline-acetyltransferase activity necessitated by increased cholinergic input, and increased neuronal membrane turnover activity in the bilateral Hip and right PCC in the aMCI stage due to further neuronal degeneration. This is consistent with our previous observations $(30,34)$.

In previous studies, $\mathrm{tCr}$ concentrations have been considered to be stable and commonly used as an internal reference for normalization of other metabolites. Our results showed that the $\mathrm{tCr}$ concentrations had a decreasing trend from ECs to patients with aMCI. The noteworthy result of our study was that we found a significant reduction in $\mathrm{tCr}$ values in the left DT and right OLWM in aMCI participants. Some reports have also found that the $\mathrm{tCr}$ concentrations may vary in different pathological conditions $(23,35)$. The reduction in $\mathrm{tCr}$ levels represent an insufficient energy supply that may result from a defect in oxidative metabolism in impaired nerve cell (58). Together, the observations from previous studies and those from this study provided evidence that SCD plus and aMCI participants showed ultrastructural damage in the neuronal mitochondria. These results confirmed that the absolute concentration might be better than the relative ratios estimated using $\mathrm{tCr}$ as a reference.

There were five brain regions with altered metabolites, including four regions with decreased NAA (left Hip, PCC, Pr, and CNC) and one region with increased $\mathrm{mI}$ (left Hip). Similarly, the differences in NAA/mI ratios were also found in five brain regions (left Hip, left PCC, left $\mathrm{Pr}$, left OLWM, and right Hip). Both these evaluations revealed four brain areas in the left hemisphere that contained vulnerable regions involved in memory function and language skills (59-62). The different concentration of NAA between ECs and SCD plus in the left cerebral hemisphere indicated that atrophy in SCD plus could have already occurred in $\mathrm{AD}$-sensitive brain areas such as left Hip (48). The combined AUC values in distinguishing SCD plus from ECs participants acquired by the concentration of NAA combined with $\mathrm{mI}$ was 0.895 , which was higher than the combined AUC values of 0.860 acquired by the $\mathrm{NAA} / \mathrm{mI}$ ratios. Compared to patients with aMCI, the concentration changes of NAA were found in the left $\mathrm{Pr}$, right Hip, right PCC, and $\mathrm{mI}$ levels in the right Hip and right PCC in SCD plus participants. Significantly different $\mathrm{NAA} / \mathrm{mI}$ ratios were observed in three brain regions (left Pr, right Hip, and right PCC) in SCD plus participants. Likewise, the combined AUC levels in distinguishing SCD plus from aMCI participants acquired by NAA combined with $\mathrm{mI}$ levels was 0.892 , which was also higher than the combined AUC values of 0.836 acquired by NAA/mI ratios. Our results further demonstrated that the absolute concentration may be superior to the metabolite ratio, which is consistent with previous ${ }^{1} \mathrm{H}$-MRS studies. The possible reason is that the absolute concentration could offer more accurate information than the metabolite ratio reflecting brain metabolite changes.

In addition, we observed AVLT-De scores were significantly associated with the NAA values in the left Hip and left PCC in SCD plus group. Only the AVLT-De scores were positively correlated with $\mathrm{NAA} / \mathrm{mI}$ ratio in the left Hip. We speculated that the main reason for the effect on the two brain regions could have been the abnormal changes of NAA, while $\mathrm{mI}$ may have had a synergistic effect. Our correlation results were also in favor of the idea that the NAA change might be the best predictor of cognitive impairment scores, especially in the extremely early phase of $\mathrm{AD}$.

This study has some limitations. Firstly, as a crosssectional study, the samples of patients with SCD plus are small. A study with a larger sample size with a multicenter and longitudinal design is acquired to seek the early neuroimaging markers for $\mathrm{AD}(63,64)$. Secondly, in order to fully elucidate the $\mathrm{AD}$ continuous spectrum, $\mathrm{AD}$ individuals need to be included in the subsequent research. Thirdly, according to the features of SCD plus, the inclusion criteria for patients with SCD plus usually met more than three (13). Although the NAA and mI levels are emerging as the most useful MRS markers in $\mathrm{AD}$ since this measure could be strongly associated with the existence of potential amyloidosis and neurodegeneration, our study lacked the completeness of testing the status of ApoE $\varepsilon 4$ (65), tau protein, or $\beta$-amyloid. Incorporating the examinations of these risk factors may be crucial for SCD plus detection. Moreover, although ${ }^{1} \mathrm{H}-\mathrm{MVS}$ could 
obtain a wider range of multiple single voxel spectra, reduce the partial volume effect, and reflect the distribution of metabolite concentrations throughout multi-dimensional data acquisition, certain limitations of ${ }^{1} \mathrm{H}-\mathrm{MVS}$, such as long acquisition time, poor quality of shimming, inadequate water peak suppression, and susceptibility alterations related to air and bone, make it hard to acquire highquality spectra. Besides, the study only used MRS technique for evaluation. Multi-modal MR imaging methods would yield a comprehensive understanding to elucidate the pathophysiologic mechanisms of participants with SCD plus by structural and functional MRI (66-68). Finally, we did not register the MRS data to utilize tissue segmentation results from a high-resolution anatomical image to calculate the white matter, gray matter, or CSF fraction within the VOIs; therefore, a variance source should be taken into account for correcting the metabolite concentrations more accurately.

\section{Conclusions}

In conclusion, our findings indicated that quantitative ${ }^{1} \mathrm{H}$-SVS and ${ }^{1} \mathrm{H}$-MVS could offer useful information to detect the feature of the alterative brain neurometabolite levels in patients with SCD plus. The use of absolute concentration rather than ratios might provide more valuable information regarding alternative metabolites associated with AD. NAA may be one of the earliest potential neuroimaging markers at this very early stage. Changes in brain metabolite concentrations in the PCC and Hip appeared to represent a noninvasive, effective, and potentially useful neuroimaging marker of the preclinical stage of $\mathrm{AD}$.

\section{Acknowledgments}

Funding: This study was supported by the National Natural Science Foundation of China (Grant Number: 81671660), Natural Science Foundation of Guangdong Province (Grant Number: 2018A030310154), Medical Science Research Foundation of Guangdong Province (Grant Number: A2017532), Science and Technology Project of Shenzhen (Grant Number: JCYJ20170307144338570), Clinical Research Startup Program of Southern Medical University by High-level University Construction Funding of Guangdong Provincial Department of Education (Grant Number: LC2016PY061) and Shenzhen Hospital of Southern Medical University, Research Promotion Funds for the Key Discipline Construction Program (Grant Number: ZDXKKYTS010 \& ZDXKKYTS011).

\section{Footnote}

Conflicts of Interest: All authors have completed the ICMJE uniform disclosure form (available at http://dx.doi. org/10.21037/qims-20-1254). The authors have no conflicts of interest to declare.

Ethical Statement: The authors are accountable for all aspects of the work in ensuring that questions related to the accuracy or integrity of any part of the work are appropriately investigated and resolved. The study was conducted in accordance with the Declaration of Helsinki (as revised in 2013) and approved by the Ethics Committee of the second Affiliated Hospital of Shantou University Medical College. Written informed consent was obtained from all participants or his/her legal guardians.

Open Access Statement: This is an Open Access article distributed in accordance with the Creative Commons Attribution-NonCommercial-NoDerivs 4.0 International License (CC BY-NC-ND 4.0), which permits the noncommercial replication and distribution of the article with the strict proviso that no changes or edits are made and the original work is properly cited (including links to both the formal publication through the relevant DOI and the license). See: https://creativecommons.org/licenses/by-nc-nd/4.0/.

\section{References}

1. Tandetnik C, Farrell MT, Cary MS, Cines S, Emrani S, Karlawish J, Cosentino S. Ascertaining Subjective Cognitive Decline: A Comparison of Approaches and Evidence for Using an Age-Anchored Reference Group. J Alzheimers Dis 2015;48:S43-55.

2. Jonker C, Geerlings MI, Schmand B. Are memory complaints predictive for dementia? A review of clinical and population-based studies. Int J Geriatr Psychiatry 2000;15:983-91.

3. Mitchell AJ, Beaumont H, Ferguson D, Yadegarfar M, Stubbs B. Risk of dementia and mild cognitive impairment in older people with subjective memory complaints: metaanalysis. Acta Psychiatr Scand 2014;130:439-51.

4. Peter J, Scheef L, Abdulkadir A, Boecker H, Heneka M, Wagner M, Koppara A, Klöppel S, Jessen F, Alzheimer's Disease Neuroimaging Initiative. Gray matter atrophy 
pattern in elderly with subjective memory impairment. Alzheimers Dement 2014;10:99-108.

5. Saykin AJ, Wishart HA, Rabin LA, Santulli RB, Flashman LA, West JD, McHugh TL, Mamourian AC. Older adults with cognitive complaints show brain atrophy similar to that of amnestic MCI. Neurology 2006;67:834-42.

6. Wen Q, Mustafi SM, Li J, Risacher SL, Tallman E, Brown SA, West JD, Harezlak J, Farlow MR, Unverzagt FW, Gao S, Apostolova LG, Saykin AJ, Wu YC. White matter alterations in early-stage Alzheimer's disease: A tractspecific study. Alzheimers Dement (Amst) 2019;11:576-87.

7. Jeong HS, Park JS, Song IU, Chung YA, Rhie SJ. Changes in cognitive function and brain glucose metabolism in elderly women with subjective memory impairment: a 24-month prospective pilot study. Acta Neurol Scand 2017;135:108-14.

8. Snitz BE, Weissfeld LA, Lopez OL, Kuller LH, Saxton J, Singhabahu DM, Klunk WE, Mathis CA, Price JC, Ives DG, Cohen AD, McDade E, Dekosky ST. Cognitive trajectories associated with -amyloid deposition in the oldest-old without dementia. Neurology 2013;80:1378-84.

9. Shu N, Wang X, Bi Q, Zhao T, Han Y. Disrupted Topologic Efficiency of White Matter Structural Connectome in Individuals with Subjective Cognitive Decline. Radiology 2018;286:229-38.

10. Reisberg B, Shao Y, Moosavi M, Kenowsky S, Vedvyas A, Marsh K, Bao J, Buj M, Torossian C, Kluger A, Vedvyas G, Oo T, Malik F, Arain F, Masurkar AV, Wisniewski T. Psychometric Cognitive Decline Precedes the Advent of Subjective Cognitive Decline in the Evolution of Alzheimer's Disease. Dement Geriatr Cogn Disord 2020;49:16-21.

11. Yan T, Wang W, Yang L, Chen K, Chen R, Han Y. Rich club disturbances of the human connectome from subjective cognitive decline to Alzheimer's disease. Theranostics 2018;8:3237-55.

12. Perrotin A, La Joie R, de La Sayette V, Barré L, Mézenge F, Mutlu J,Guilloteau D, Egret S, Eustache F, Chételat G. Subjective cognitive decline in cognitively normal elders from the community or from a memory clinic: Differential affective and imaging correlates. Alzheimers Dement 2017; 13:550-60.

13. Jessen F, Amariglio RE, van Boxtel M, Breteler M, Ceccaldi M, Chételat G, et al. Subjective Cognitive Decline Initiative (SCD-I) Working Group. A conceptual framework for research on subjective cognitive decline in preclinical Alzheimer's disease. Alzheimers Dement 2014;10:844-52.
14. Hao L, Sun Y, Li Y, Wang J, Wang Z, Zhang Z, Wei Z, Gao G, Jia J, Xing Y, Han Y. Demographic characteristics and europsychological assessments of subjective cognitive decline (SCD) (plus). Ann Clin Transl Neurol 2020;7:1002-12.

15. Sánchez-Benavides G, Grau-Rivera $O$, Suárez-Calvet $M$, Minguillon C, Cacciaglia R, Gramunt N, Falcon C; ALFA Study, Gispert JD, Molinuevo JL. Brain and cognitive correlates of subjective cognitive decline-plus features in a population-based cohort. Alzheimers Res Ther 2018;10:123.

16. Fayed N, Modrego PJ, Salinas GR, Gazulla J. Magnetic resonance imaging based clinical research in Alzheimer's disease. J Alzheimers Dis 2012;31:S5-S18.

17. Moffett JR, Ross B, Arun P, Madhavarao CN, Namboodiri AM. N-Acetylaspartate in the CNS: from neurodiagnostics to neurobiology. Prog Neurobiol 2007;81:89-131.

18. Voevodskaya O, Poulakis K, Sundgren P, van Westen D, Palmqvist S, Wahlund LO, Stomrud E, Hansson O, Westman E; Swedish BioFINDER Study Group. Brain myoinositol as a potential marker of amyloidrelated pathology: A longitudinal study. Neurology 2019;92:e395-e405.

19. Catani M, Cherubini A, Howard R, Tarducci R, Pelliccioli GP, Piccirilli M, Gobbi G, Senin U, Mecocci P. (1)H-MR spectroscopy differentiates mild cognitive impairment from normal brain aging. Neuroreport 2001;12:2315-7.

20. Waldman AD, Rai GS. The relationship between cognitive impairment and in vivo metabolite ratios in patients with clinical Alzheimer's disease and vascular dementia: a proton magnetic resonance spectroscopy study. Neuroradiology 2003;45:507-12.

21. Wang H, Tan L, Wang HF, Liu Y, Yin RH, Wang WY, Chang XL, Jiang T, Yu JT. Magnetic Resonance Spectroscopy in Alzheimer's Disease: Systematic Review and Meta-Analysis. J Alzheimers Dis 2015;46:1049-70.

22. Kantarci K, Weigand SD, Przybelski SA, Shiung MM, Whitwell JL, Negash S, Knopman DS, Boeve BF, O'Brien PC, Petersen RC, Jack CR Jr. Risk of dementia in MCI: combined effect of cerebrovascular disease, volumetric MRI, and 1H MRS. Neurology 2009;72:1519-25.

23. Gasparovic C, Prestopnik J, Thompson J, Taheri S, Huisa B, Schrader R, Adair JC, Rosenberg GA. 1H-MR spectroscopy metabolite levels correlate with executive function in vascular cognitive impairment. J Neurol Neurosurg Psychiatry 2013;84:715-21.

24. Xu S, Zhu W, Wan Y, Wang J, Chen X, Pi L, Lobo MK, Ren B, Ying Z, Morris M, Cao Q. Decreased Taurine 
and Creatine in the Thalamus May Relate to Behavioral Impairments in Ethanol-Fed Mice: A Pilot Study of Proton Magnetic Resonance Spectroscopy. Mol Imaging 2018;17:1536012117749051.

25. Jessen F, Gür O, Block W, Ende G, Frölich L, Hammen T, Wiltfang J, Kucinski T, Jahn H, Heun R, Maier W, Kölsch H, Kornhuber J, Träber F. A multicenter (1)H-MRS study of the medial temporal lobe in AD and MCI. Neurology 2009;72:1735-40.

26. Franczak M, Prost RW, Antuono PG, Mark LP, Jones JL, Ulmer JL. Proton magnetic resonance spectroscopy of the hippocampus in patients with mild cognitive impairment: a pilot study. J Comput Assist Tomogr 2007;31:666-70.

27. Chawla S, Wang S, Moore P, Woo JH, Elman L, McCluskey LF, Melhem ER, Grossman M, Poptani H. Quantitative proton magnetic resonance spectroscopy detects abnormalities in dorsolateral prefrontal cortex and motor cortex of patients with frontotemporal lobar degeneration. J Neurol 2010;257:114-21.

28. Modrego PJ, Fayed N, Pina MA. Conversion from mild cognitive impairment to probable Alzheimer's disease predicted by brain magnetic resonance spectroscopy. Am J Psychiatry 2005;162:667-75.

29. Modrego PJ, Fayed N, Sarasa M. Magnetic resonance spectroscopy in the prediction of early conversion from amnestic mild cognitive impairment to dementia: a prospective cohort study. BMJ Open 2011;1:e00007.

30. Yang ZX, Huo SS, Cheng XF, Xu ZF, Cao Z, Zeng JX, Xiao YY, You KZ, Chen W, Liu YY, Wu RH. Quantitative multivoxel proton MR spectroscopy study of brain metabolites in patients with amnestic mild cognitive impairment: a pilot study. Neuroradiology 2012;54:451-8.

31. Zimny A, Szewczyk P, Trypka E, Wojtynska R, Noga L, Leszek J, Sasiadek M. Multimodal imaging in diagnosis of Alzheimer's disease and amnestic mild cognitive impairment: value of magnetic resonance spectroscopy, perfusion, and diffusion tensor imaging of the posterior cingulate region. J Alzheimers Dis 2011;27:591-601.

32. Tumati S, Martens S, Aleman A. Magnetic resonance spectroscopy in mild cognitive impairment: systematic review and meta-analysis. Neurosci Biobehav Rev 2013;37:2571-86.

33. Li H, Xu H, Zhang Y, Guan J, Zhang J, Xu C, Shen Z, Xiao B, Liang C, Chen K, Zhang J, Wu R. Differential neurometabolite alterations in brains of medication-free individuals with bipolar disorder and those with unipolar depression: a two-dimensional proton magnetic resonance spectroscopy study. Bipolar Disord 2016;18:583-90.

34. Liu YY, Yang ZX, Shen ZW, Xiao YY, Cheng XF, Chen W, Chen YW, Wu RH. Magnetic Resonance Spectroscopy Study of Amnestic Mild Cognitive Impairment and Vascular Cognitive Impairment With No Dementia. Am J Alzheimers Dis Other Demen 2014;29:474-81.

35. Zhang Z, Wang Y, Shen Z, Yang Z, Li L, Chen D, Yan G, Cheng X, Shen Y, Tang X, Hu W, Wu R. The Neurochemical and Microstructural Changes in the Brain of Systemic Lupus Erythematosus Patients: A Multimodal MRI Study. Sci Rep 2016;6:19026.

36. Folstein MF, Folstein SE, McHugh PR. "Mini-mental state". A practical method for grading the cognitive state of patients for the clinician. J Psychiatr Res 1975;12:189-98.

37. Lu J, Li D, Li F, Zhou A, Wang F, Zuo X, Jia XF, Song $\mathrm{H}$, Jia J. Montreal cognitive assessment in detecting cognitive impairment in Chinese elderly individuals: a population-based study. J Geriatr Psychiatry Neurol 2011;24:184-90.

38. Morris JC. The Clinical Dementia Rating (CDR): current version and scoring rules. Neurology 1993;43:2412-4.

39. Reisberg B, Ferris SH, de Leon MJ, Crook T. The Global Deterioration Scale for assessment of primary degenerative dementia. Am J Psychiatry 1982;139:1136-9.

40. Xu Y, Chen K, Zhao Q, Li F, Guo Q. Short-term delayed recall of auditory verbal learning test provides equivalent value to long-term delayed recall in predicting MCI clinical outcomes: A longitudinal follow-up study. Appl Neuropsychol Adult 2020;27:73-81.

41. Barberger-Gateau P, Commenges D, Gagnon M, Letenneur L, Sauvel C, Dartigues JF. Instrumental activities of daily living as a screening tool for cognitive impairment and dementia in elderly community dwellers. J Am Geriatr Soc 1992;40:1129-34.

42. Larson DB, Lyons JS, Bareta JC, Burns BJ, Blazer DG, Goldstrom ID. The construct validity of the ischemic score of Hachinski for the detection of dementias. J Neuropsychiatry Clin Neurosci 1989;1:181-7.

43. Worboys M. The Hamilton Rating Scale for Depression: The making of a "gold standard" and the unmaking of a chronic illness, 1960-1980. Chronic Illn 2013;9:202-19.

44. Petersen RC, Doody R, Kurz A, Mohs RC, Morris JC, Rabins PV, Ritchie K, Rossor M, Thal L, Winblad B. Current concepts in mild cognitive impairment. Arch Neurol 2001;58:1985-92. 
45. Mitolo M, Stanzani-Maserati M, Capellari S, Testa C, Rucci P, Poda R, Oppi F, Gallassi R, Sambati L, Rizzo G, Parchi P, Evangelisti S, Talozzi L, Tonon C, Lodi R, Liguori R. Predicting conversion from mild cognitive impairment to Alzheimer's disease using brain 1H-MRS and volumetric changes: A two-year retrospective followup study. Neuroimage Clin 2019;23:101843.

46. Zhu X, Schuff N, Kornak J, Soher B, Yaffe K, Kramer JH, Ezekiel F, Miller BL, Jagust WJ, Weiner MW. Effects of Alzheimer disease on fronto-parietal brain $\mathrm{N}$-acetyl aspartate and myo-inositol using magnetic resonance spectroscopic imaging. Alzheimer Dis Assoc Disord 2006;20:77-85.

47. Waragai M, Moriya M, Nojo T. Decreased N-Acetyl Aspartate/Myo-Inositol Ratio in the Posterior Cingulate Cortex Shown by Magnetic Resonance Spectroscopy May Be One of the Risk Markers of Preclinical Alzheimer's Disease: A 7-Year Follow-Up Study. J Alzheimers Dis 2017;60:1411-27.

48. Perrotin A, de Flores R, Lamberton F, Poisnel G, La Joie $\mathrm{R}$, de la Sayette V, Mézenge F, Tomadesso C, Landeau B, Desgranges B, Chételat G. Hippocampal Subfield Volumetry and 3D Surface Mapping in Subjective Cognitive Decline. J Alzheimers Dis 2015;48:S141-50.

49. Watanabe T, Shiino A, Akiguchi I. Absolute quantification in proton magnetic resonance spectroscopy is useful to differentiate amnesic mild cognitive impairment from Alzheimer's disease and healthy aging. Dement Geriatr Cogn Disord 2010;30:71-7.

50. Heales SJ, Davies SE, Bates TE, Clark JB. Depletion of brain glutathione is accompanied by impaired mitochondrial function and decreased $\mathrm{N}$-acetyl aspartate concentration. Neurochem Res 1995;20:31-8.

51. Liang S, Huang J, Liu W, Jin H, Li L, Zhang X, Nie B, Lin R, Tao J, Zhao S, Shan B, Chen L. Magnetic resonance spectroscopy analysis of neurochemical changes in the atrophic hippocampus of APP/PS1 transgenic mice. Behav Brain Res 2017;335:26-31.

52. Glodzik L, Sollberger M, Gass A, Gokhale A, Rusinek H, Babb JS, Hirsch JG, Amann M, Monsch AU, Gonen O. Global $\mathrm{N}$-acetylaspartate in normal subjects, mild cognitive impairment and Alzheimer's disease patients. J Alzheimers Dis 2015;43:939-47.

53. Madhavarao CN, Chinopoulos C, Chandrasekaran K, Namboodiri MA. Characterization of the $\mathrm{N}$-acetylaspartate biosynthetic enzyme from rat brain. J Neurochem 2003;86:824-35.

54. Schofield E, Kersaitis C, Shepherd CE, Kril JJ,
Halliday GM. Severity of gliosis in Pick's disease and frontotemporal lobar degeneration: tau-positive glia differentiate these disorders. Brain 2003;126:827-40.

55. Voevodskaya O, Sundgren PC, Strandberg O, Zetterberg H, Minthon L, Blennow K, Wahlund LO, Westman E, Hansson O; Swedish BioFINDER study group. Myoinositol changes precede amyloid pathology and relate to APOE genotype in Alzheimer disease. Neurology 2016;86:1754-61.

56. Majós C, Alonso J, Aguilera C, Serrallonga M, Acebes JJ, Arús C, Gili J. Adult primitive neuroectodermal tumor: proton MR spectroscopic findings with possible application for differential diagnosis. Radiology 2002;225:556-66.

57. Huang C, Wahlund LO, Svensson L, Winblad B, Julin P. Cingulate cortex hypoperfusion predicts Alzheimer's disease in mild cognitive impairment. BMC Neurol 2002;2:9.

58. Watanabe T, Shiino A, Akiguchi I. Absolute quantification in proton magnetic resonance spectroscopy is superior to relative ratio to discriminate Alzheimer's disease from Binswanger's disease. Dement Geriatr Cogn Disord 2008;26:89-100.

59. Corballis MC. Evolution of cerebral asymmetry. Prog Brain Res 2019;250:153-78.

60. Keller SS, Roberts N, Baker G, Sluming V, Cezayirli E, Mayes A, Eldridge P, Marson AG, Wieshmann UC. A voxel-based asymmetry study of the relationship between hemispheric asymmetry and language dominance in Wada tested patients. Hum Brain Mapp 2018;39:3032-45.

61. Watanabe T, Shiino A, Akiguchi I. Hippocampal metabolites and memory performances in patients with amnestic mild cognitive impairment and Alzheimer's disease. Neurobiol Learn Mem 2012;97:289-93.

62. Zhou IY, Ding AY, Li Q, McAlonan GM, Wu EX. Magnetic resonance spectroscopy reveals $\mathrm{N}$-acetylaspartate reduction in hippocampus and cingulate cortex after fear conditioning. Psychiatry Res 2012;204:178-83.

63. Jessen F, Spottke A, Boecker H, Brosseron F, Buerger $\mathrm{K}$, Catak C, et al. Design and first baseline data of the DZNE multicenter observational study on predementia Alzheimer's disease (DELCODE). Alzheimers Res Ther 2018;10:15.

64. Modrego PJ, Fayed N. Longitudinal magnetic resonance spectroscopy as marker of cognitive deterioration in mild cognitive impairment. Am J Alzheimers Dis Other Demen 2011;26:631-6.

65. Zhang C, Kong M, Wei H, Zhang H, Ma G, Ba M; 
Alzheimer's Disease Neuroimaging Initiative. The effect of ApoE $\varepsilon 4$ on clinical and structural MRI markers in prodromal Alzheimer's disease. Quant Imaging Med Surg 2020;10:464-74.

66. Costigan AG, Umla-Runge K, Evans CJ, Hodgetts CJ, Lawrence AD, Graham KS. Neurochemical correlates of scene processing in the precuneus/posterior cingulate cortex: A multimodal fMRI and 1H-MRS study. Hum Brain Mapp 2019;40:2884-98.

67. Wei H, Kong M, Zhang C, Guan L, Ba M; for Alzheimer's
Disease Neuroimaging Initiative*. The structural MRI markers and cognitive decline in prodromal Alzheimer's disease: a 2-year longitudinal study. Quant Imaging Med Surg 2018;8:1004-19.

68. Yang Z, Rong Y, Cao Z, Wu Y, Zhao X, Xie Q, Luo M, Liu Y. Microstructural and Cerebral Blood Flow Abnormalities in Subjective Cognitive Decline Plus: Diffusional Kurtosis Imaging and Three-Dimensional Arterial Spin Labeling Study. Front Aging Neurosci 2021;13:625843.

Cite this article as: Yang Z, Wan X, Zhao X, Rong Y, Wu Y, Cao Z, Xie Q, Luo M, Liu Y. Brain neurometabolites differences in individuals with subjective cognitive decline plus: a quantitative single- and multi-voxel proton magnetic resonance spectroscopy study. Quant Imaging Med Surg 2021;11(9):4074-4096. doi: 10.21037/qims-20-1254 


\section{Supplementary material 1}

\section{Supplementary file 1 (bow to obtain Pflie from MRI bost)}

\section{[Open Window 1]}

1. cd researchdata/ [Enter]

2. 1s [Enter]

3. cd (current folder, for example: 20190628/) [Enter]

4. mkdir (create file name, for example: abc) [Enter]

5. cd (current file name, for example: abc) [Enter]

6. pwd [Enter]

Then we get the created folder path

\section{[Open Window 2]}

1. ftp 10.0.12.110 [Enter] (Note: This is an IP address which corresponds to different hosts)

2. sdc [Enter]

3. adw 2.0 [Enter] (Note: This is a password which corresponds to different hosts)

4. bin [Enter]

5. lcd (copy the folder path from Window 1) [Enter]

6. cd /usr/g/mrraw [Enter]

7. 1s -tl [Enter] (Note: It shows recent pfiles which we can select and copy these according to experiment time)

8. get P04068.7 (pfile name) [Enter] (Note: Then the pfile was successfully obtained. We repeat the command "get" to obtain the pfile)

9. exit [Enter]

Then we exit window 2 and return to window 1 .

\section{Copy pfile to $\mathrm{U}$ disk from usb path}

1. cd..

2. su root [Enter]

3. operator [Enter]

4. fdisk -1 [Enter] (Then we obtain the path of usb, for example: /del/usb)

5. mount -t vfat (for example: /del/usb) /mnt/usbdisk [Enter]

6. сp -r (The name of the folder created in window 1, for example: abc) /mnt/usbdisk [Enter] Note: You must confirm that window 1 has entered the abc folder before executing this command.

7. cd /mnt/usbdisk [Enter]

8. 1s [Enter]

9. cd / [Enter]

10. umount /mnt/usbdisk [Enter]

11. exit [Enter]

Then we copy the pfile to $U$ disk successfully. 


\section{Supplementary material 2}

\section{Supplementary file 2 (the method to analysis MRS)}

The analysis step for single-voxel magnetic resonance spectroscopy using LCModel

1. Turn on the lab computer, enter the username and password

2. Open the virtual machine on the desktop (VMware), right-click on the username (for example: administrator) in the current mode, enter password (for example: dell123)

3. Double-click the terminal, then input cd. lcmodel [enter]

4. Click ./lcmgui [enter]

5. Click the selected profile

6. Select a pile from the path $/ u s r / g / s p e c t r o / d a t a / a b c / 20190806$

7. Advanced settings: change control defaut file: select 3T, click OK

8. Click change basis, then selcet press_te35_3t_gsh_v3.basis

9. Click LCModel, then start to analysis results

\section{The analysis step for multi-voxel magnetic resonance spectroscopy using LCModel and SAGE software}

1. Turn on the lab computer, enter the username and password;

2. Open the virtual machine on the desktop (VMware), right-click on the username (for example: administrator) in the current mode, enter password (for example: dell123)

3. Double-click the terminal, then input cd. lcmodel [enter]

4. Click terminal: SAGE

5. Import Pflie, selcet the path mnt/hgfs/libui /usr/g/spectro/data/abc/20190806

6. Open a Pfile and reconstruct it: recons CSI reconstruct

7. Analysis by LCModel

8. Select the path of LCModel: SDDEGE:999; SDDEGP:1

9. Enter PPMST:4.0, PPMEND:0.2

10. Select calibration factor: First, the NAA value from single-voxel proton magnetic resonance spectroscopy (NAAsvs) is processed obtain in a selected volume of interest. The correction factor of multi-voxel proton magnetic resonance spectroscopy filled with 1, and then NAA value from multi-voxel proton magnetic resonance spectroscopy (NAAmvs) is obtained. The ratio NAAmvs/NAAsvs corresponding the same volume of interest was acquired, and it is the final calibration factor.

11. Then run Basis set: press-te35-3t-gsh-v3-GE.basis

12. Start to analysis results 


\section{Supplementary material 3}

\section{Supplementary file 3 (the basic process flow of the RF algorithm)}

setwd('G: \\WORK-MBY\\data classification management \\revision manuscript')

library(randomForest)

library(readxl)

library(sqldf)

library(stringr)

library(pROC)

library(ROCR)

library(caret)

library(dplyr)

str2num <- function(data) \{as.numeric(as.character(data))\}

df1 $<-$ read_excel('data.xlsx', sheet $=1$ )

df2 $<-$ read_excel('data.xlsx', sheet $=2$ )

\#\#model1, ratio1==========================================="

\#\# use sex, age, education, variable $\mathrm{NAA} / \mathrm{mI}$

$\mathrm{df}<-\mathrm{df} 1$

df\$grp $<-$ ifelse(df\$grp == 'ECs', 0, 1)

df\$gender $<-$ factor(df\$gender)

df[, str_detect(names(df), '_n\$I_im\$I_m\$')] <- NULL

set.seed(22)\#\# Set random seeds

\#\# 65\% for train

\#\# 35\% for test

train_index <- sample $\left(1\right.$ :nrow $(\mathrm{df}), 0.65{ }^{*}$ nrow $(\mathrm{df})$, replace $\left.=\mathrm{F}\right)$

train_df <- df[train_index, ]

test_df $<-$ df [-train_index, ]

rf_fit $<$ - randomForest $($ grp $~$. , data $=$ train_df, ntree $=35$, type $=$ 'regression', mtry $=4$, importance $=\mathrm{T}$ )

imp $<$ - importance(rf_fit)

imp $<-$ as.data.frame(imp)

imp\$vname <- row.names(imp)

names(imp) <- c('inci_mse', 'inc_node_purity', 'vname')

imp <- imp[, c('vname', 'inci_mse')]

imp $<-$ sqldf('select * from imp order by inci_mse desc')

Imp \#\# use test test

pred $<-$ predict(rf_fit, test_df, type = 'response')

train_pred $<$ - predict(rf_fit, train_df, type = 'response')

test_pred $<-$ pred

pred $<-$ prediction(pred, as.factor(test_df $\$$ grp))

perf <- performance(pred, 'tpr', 'fpr')

test_auc <- unlist(slot(performance(pred, 'auc'), 'y.values'))

test_auc \#\# the optimal segmentation point was found according to the model fitting results

train_cut_off $<-$ roc $($ response $=$ train_df\$grp, predictor $=$ train_pred $)$

train_cut_df <- cbind(train_cut_off\$thresholds, train_cut_off\$sensitivities + train_cut_off\$specificities)

best_cut_off $<-\operatorname{subset}(\mathrm{e}, \mathrm{e}[, 2]==\max (\mathrm{e}[, 2]))[, 1]$

\#\# predictive classification was obtained according to segmentation points

pred_class $<-$ ifelse(pred2 $>=$ best_cut_off, 1,0 )

xtab $<-$ table(pred_class, test_df\$grp) 
confusionMatrix (xtab, positive = '1')

cut_off $<-$ roc $($ response $=$ test_df $\$$ grp, predictor $=$ test_pred $)$

$\operatorname{par}(\operatorname{mgp}=\mathrm{c}(2,0.8,0)$, oma $=\mathrm{c}(0,0,0,0))$

$\operatorname{plot}(1$ - cut_off\$specificities, cut_off\$sensitivities, type = 'l', xlab = '1 - Specificity', ylab = 'Sensitivity', col = 'black', lwd = 2, bty $=$ 'l', font.lab $=2$, main $=$ 'ROC Curve', cex.lab $=1.6$, font $=2$ )

lines $(\mathrm{c}(0,1), \mathrm{c}(0,0)$, lty $=2)$

lines $(\mathrm{c}(1,1), \mathrm{c}(0,1)$, lty $=2)$

lines $(\mathrm{c}(0,1), \mathrm{c}(0,1)$, lty $=2)$

text $(0.5,0.6$, paste('AUC = ', round(test_auc, 2), sep = "), font = 2, cex = 1.2)

imp\$vname

imp <- filter(imp, str_detect(vname, '_nm\$') \%in\% c(T))

imp\$x $<-$ nrow(imp):1imp

imp\$label <- c('L-Hip(NAA/ml)', 'R-Hip(NAA/ml)', 'L-Pr(NAA/ml)', 'L-PCC(NAA/ml)', 'L-OLWM(NAA/ml)')

$\operatorname{par}(\mathrm{oma}=\mathrm{c}(0,4,0,0))$

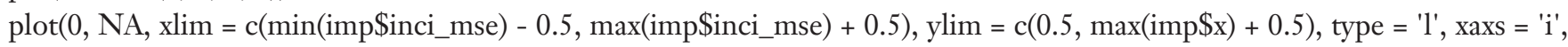

yaxs = 'i', bty = 'l', xlab = 'Importance measure $(\%$ IncMSE)', ylab = ", yaxt = 'n', font.lab = 2)

for $(\mathrm{r}$ in 1:nrow(imp))\{lines(c(min(imp\$inci_mse) - 0.5, imp\$inci_mse[r]), rep(imp\$x $[\mathrm{r}], 2), \mathrm{lwd}_{\mathrm{w}}=1,1 \mathrm{ty}=2$, xaxt = 'none')

points(imp\$inci_mse[r], imp\$x[r], pch $=19, \mathrm{col}=$ 'red', cex $=1.6)\}$

axis(side $=1$, tck $=-0.01$, font $=2$ )

axis $($ side $=2$, at $=$ imp\$x, labels $=$ imp\$label, las $=2$, font $=2)$

\#\#900*630

\#\#mode1, concentration1

\#\# use sex, age, education, variable $\mathrm{NAA} / \mathrm{mI}$

$\mathrm{df}<-\mathrm{df} 1$

df\$grp $<-$ ifelse (df\$grp == 'ECs', 0, 1)

df\$gender $<-$ factor(df\$gender)

df[, str_detect(names(df), '_nm\$')] <- NULL

set.seed(27)\#\# Set random seeds

\#\#65\% for train

\#\#35\% for test

train_index <- sample(1:nrow(df), $0.65{ }^{*}$ nrow $(\mathrm{df})$, replace $\left.=\mathrm{F}\right)$

train_df <- df[train_index, ]

test_df $<-$ df [-train_index, ]

rf_fit $<-$ randomForest $($ grp $~ .$, data $=$ train_df, ntree $=35$, type $=$ 'regression', \# mtry $=3$, importance $=\mathrm{T})$

imp $<$ - importance(rf_fit)

imp $<-$ as.data.frame(imp)

imp\$vname $<-$ row.names(imp)

names(imp) <- c('inci_mse', 'inc_node_purity', 'vname')

imp <- imp[, c('vname', 'inci_mse')]

imp $<-$ sqldf('select * from imp order by inci_mse desc')

imp \#\# use test test

pred $<$ - predict(rf_fit, test_df, type $=$ 'response')

train_pred <- predict(rf_fit, train_df, type = 'response')

test_pred $<-$ pred

pred $<-$ prediction(pred, as.factor(test_df\$grp))

perf <- performance(pred, 'tpr', 'fpr')

test_auc <- unlist(slot(performance(pred, 'auc'), 'y.values'))

test_auc \#\#the optimal segmentation point was found according to the model fitting results 
train_cut_off $<-$ roc(response $=$ train_dfSgrp, predictor $=$ train_pred $)$

train_cut_df <- cbind(train_cut_off\$thresholds, train_cut_off\$sensitivities + train_cut_off\$specificities)

best_cut_off $<-\operatorname{subset}(\mathrm{e}, \mathrm{e}[, 2]==\max (\mathrm{e}[, 2]))[, 1]$

\#\#predictive classification was obtained according to segmentation points

pred_class $<-$ ifelse(pred2 >= best_cut_off, 1,0 )

xtab $<-$ table(pred_class, test_df\$grp)

confusionMatrix $(x t a b$, positive $=11$ ')

cut_off $<-$ roc(response $=$ test_df\$grp, predictor $=$ test_pred $)$

$\operatorname{par}(\operatorname{mgp}=\mathrm{c}(2,0.8,0)$, oma $=\mathrm{c}(0,0,0,0))$

$\operatorname{plot}(1$ - cut_off\$specificities, cut_off\$sensitivities, type = 'l', xlab = '1 - Specificity', ylab = 'Sensitivity', col = 'black', lwd = 2,

bty $=$ 'l', font.lab $=2$, main $=$ 'ROC Curve', cex.lab $=1.6$, font $=2$ )

lines $(\mathrm{c}(0,1), \mathrm{c}(0,0)$, lty $=2)$

lines $(c(1,1), c(0,1)$, lty $=2)$

lines $(c(0,1), c(0,1)$, lty $=2)$

text $(0.5,0.6$, paste('AUC = ', round(test_auc, 2$)$, sep = "), font = 2, cex = 1.2)

imp\$vname

imp <- filter(imp, str_detect(vname, '_n\$I_m\$') \%in\% c(T))

imp\$x $<-\operatorname{nrow}(\mathrm{imp}): 1$

imp

imp\$label <- c('L-Hip(NAA)', 'L-CNC(NAA)', 'L-PCC(NAA)','R-Hip(ml)', 'L-Pr(NAA)')

$\operatorname{par}($ oma $=\mathrm{c}(0,4,0,0))$

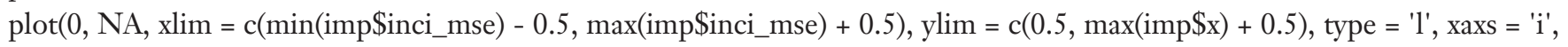

yaxs $=$ 'i', bty $=$ 'l',

xlab = 'Importance measure (\%IncMSE)', ylab = ", yaxt = 'n', font.lab = 2)

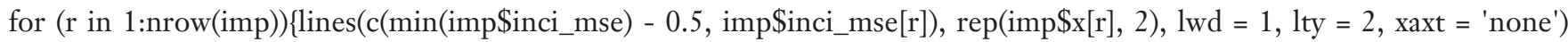

points(imp\$inci_mse[r], imp\$x[r], pch =19, col = 'red', cex $=1.6)\}$

axis $($ side $=1$, tck $=-0.01$, font $=2)$

axis $($ side $=2$, at $=$ imp\$x, labels $=$ imp\$label, las $=2$, font $=2)$

\#\#model2, ratio2

\#\# use sex, age, education, variable $\mathrm{NAA} / \mathrm{mI}$

$\mathrm{df}<-\mathrm{df} 2$

df\$grp $<-$ ifelse(df\$grp =- 'SCDplus', 0, 1)

df\$gender $<-$ factor(df\$gender)

df[, str_detect(names(df), '_n\$I_im\$I_m\$')] <- NULL

set.seed(22)\#\#Set random seeds to facilitate duplication of results

\#\#65\% for train

\#\#35\% for test

train_index <- sample(1:nrow(df), 0.65 * nrow $(\mathrm{df})$, replace $=\mathrm{F})$

train_df <- df[train_index, ]

test_df $<-$ df[-train_index, ]

rf_fit $<-$ randomForest $($ grp $~$. , data $=$ train_df, ntree $=35$, type $=$ 'regression', $\mathrm{mtry}=4$, importance $=\mathrm{T}$ )

imp $<$ - importance(rf_fit)

imp $<-$ as.data.frame(imp)

imp\$vname $<-$ row.names(imp)

names(imp) <- c('inci_mse', 'inc_node_purity', 'vname')

imp $<-$ imp[, c('vname', 'inci_mse')]

imp <- sqldf('select * from imp order by inci_mse desc')

imp 


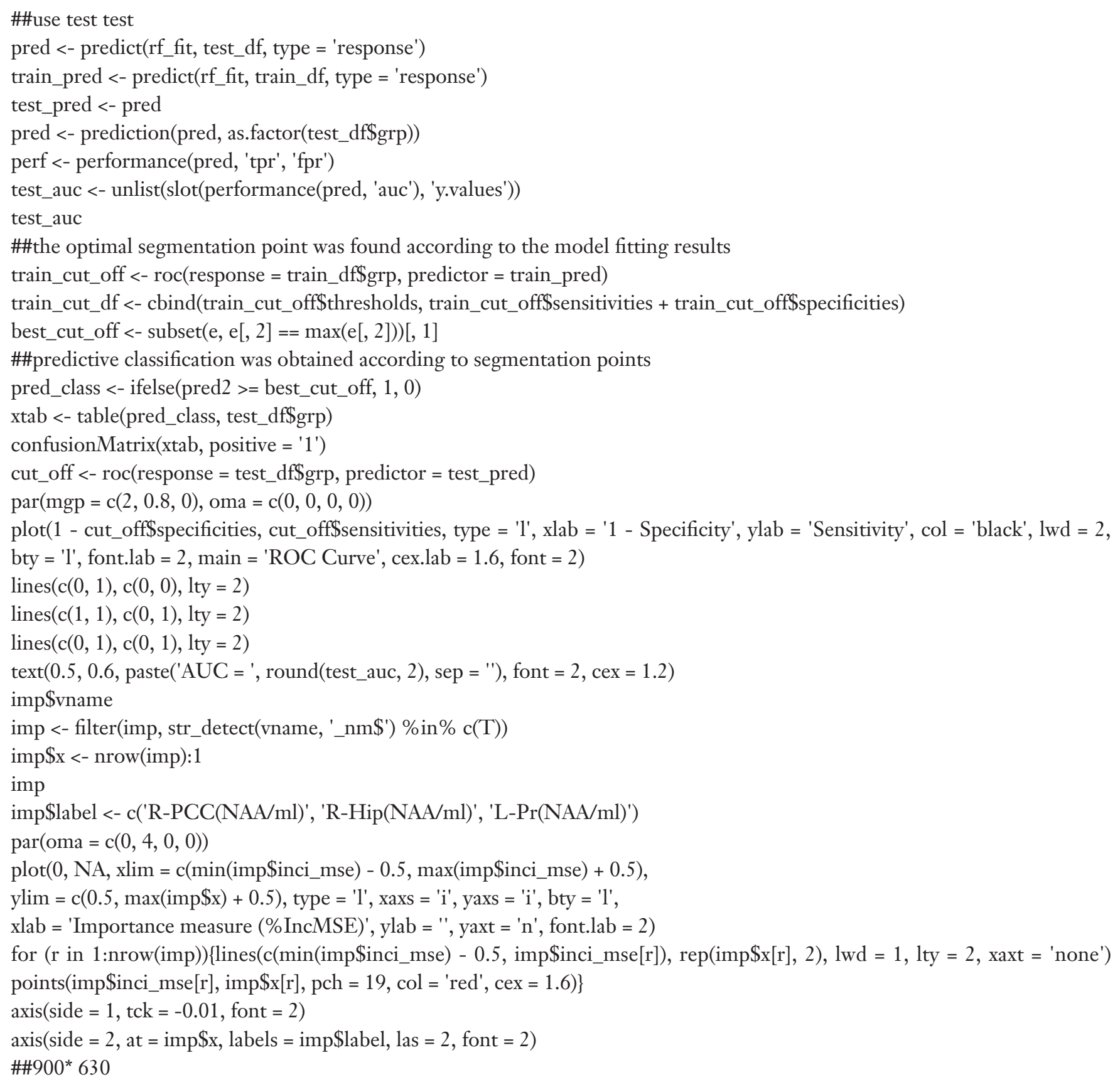


train_df $<-$ df [train_index, ]

test_df $<-$ df [-train_index, ]

rf_fit $<-$ randomForest $($ grp $~ .$, data $=$ train_df, ntree $=35$, type $=$ 'regression', \# mtry = 4, importance $=\mathrm{T}$ )

imp $<-$ importance(rf_fit)

imp $<-$ as.data.frame(imp)

imp\$vname <- row.names(imp)

names(imp) <- c('inci_mse', 'inc_node_purity', 'vname')

imp <- imp[, c('vname', 'inci_mse')]

imp $<-$ sqldf('select * from imp order by inci_mse desc')

imp

\#\# use test test

pred $<-$ predict(rf_fit, test_df, type $=$ 'response')

train_pred $<-$ predict(rf_fit, train_df, type = 'response')

test_pred $<-$ pred

pred $<-$ prediction(pred, as.factor(test_df\$grp))

perf <- performance(pred, 'tpr', 'fpr')

test_auc <- unlist(slot(performance(pred, 'auc'), 'y.values'))

test_auc

\#\#the optimal segmentation point was found according to the model fitting results

train_cut_off $<-$ roc $($ response $=$ train_df\$grp, predictor $=$ train_pred $)$

train_cut_df <- cbind(train_cut_off\$thresholds, train_cut_off\$sensitivities + train_cut_off\$specificities)

best_cut_off <- subset(e, e[, 2] == $\max (\mathrm{e}[, 2]))[, 1]$

\#\#predictive classification was obtained according to segmentation points

pred_class $<-$ ifelse(pred2 >= best_cut_off, 1,0$)$

$\mathrm{xtab}<-$ table(pred_class, test_df\$grp)

confusionMatrix $(x t a b$, positive $=$ ' 1 ')

cut_off $<-$ roc $($ response $=$ test_df $\$$ grp , predictor $=$ test_pred $)$

$\operatorname{par}(\operatorname{mgp}=\mathrm{c}(2,0.8,0)$, oma $=\mathrm{c}(0,0,0,0))$

plot $(1$ - cut_off\$specificities, cut_off\$sensitivities, type = 'l', xlab = '1 - Specificity', ylab = 'Sensitivity', col = 'black', lwd = 2,

bty = 'l', font.lab = 2, main = 'ROC Curve', cex.lab = 1.6, font $=2$ )

lines $(c(0,1), c(0,0), 1$ ty $=2)$

lines $(\mathrm{c}(1,1), \mathrm{c}(0,1)$, lty $=2)$

lines $(\mathrm{c}(0,1), \mathrm{c}(0,1), \mathrm{lty}=2)$

text $(0.5,0.6$, paste('AUC = ', round(test_auc, 2$)$, sep = "), font = 2, cex = 1.2)

imp\$vname

imp <- filter(imp, str_detect(vname, '_n\$I_m\$') \%in\% c(T))

imp\$x $<-\operatorname{nrow}(\mathrm{imp}): 1$

imp

imp\$label <- c('R-Hip(NAA)', 'R-PCC(ml)', 'R-PCC(NAA)', 'L-Hip(ml)', 'L-Pr(NAA)')

$\operatorname{par}($ oma $=\mathrm{c}(0,4,0,0))$

$\operatorname{plot}(0, \mathrm{NA}, \mathrm{xlim}=\mathrm{c}(\min ($ imp\$inci_mse $)-0.5, \max ($ imp\$inci_mse $)+0.5)$,

ylim $=c(0.5, \max (i m p \$ x)+0.5)$, type = 'l', xaxs = 'i', yaxs = 'i', bty = 'l',

xlab = 'Importance measure (\%IncMSE)', ylab = ", yaxt = 'n', font.lab = 2)

for ( $\mathrm{r}$ in 1:nrow(imp) $)\{\operatorname{lines}(\mathrm{c}(\mathrm{min}(\mathrm{imp} \$$ inci_mse $)$ - 0.5, imp\$inci_mse[r]),

$\operatorname{rep}(\operatorname{imp} \$ x[r], 2), 1 w d=1,1$ ty = 2, xaxt = 'none') points(imp\$inci_mse $[r]$, imp\$x $[r], p c h=19$, col = 'red', cex = 1.6)\}

axis(side $=1$, tck $=-0.01$, font $=2$ )

axis $($ side $=2$, at $=$ imp\$x, labels $=$ imp\$label, las $=2$, font $=2)$ 


\section{Supplementary material 4}

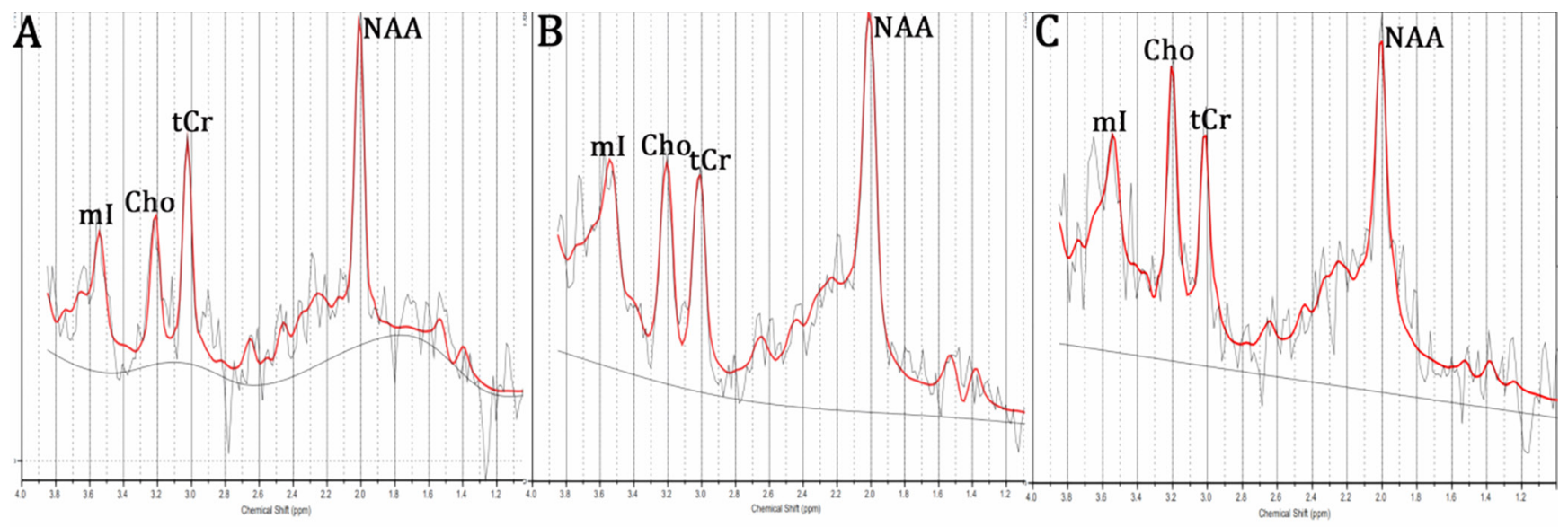

Figure S1 Examples of attained spectra from the left hippocampus. A = elderly controls, B = subjective cognitive decline plus, $\mathrm{C}=$ amnestic mild cognitive impairment.

\section{Supplementary material 5}

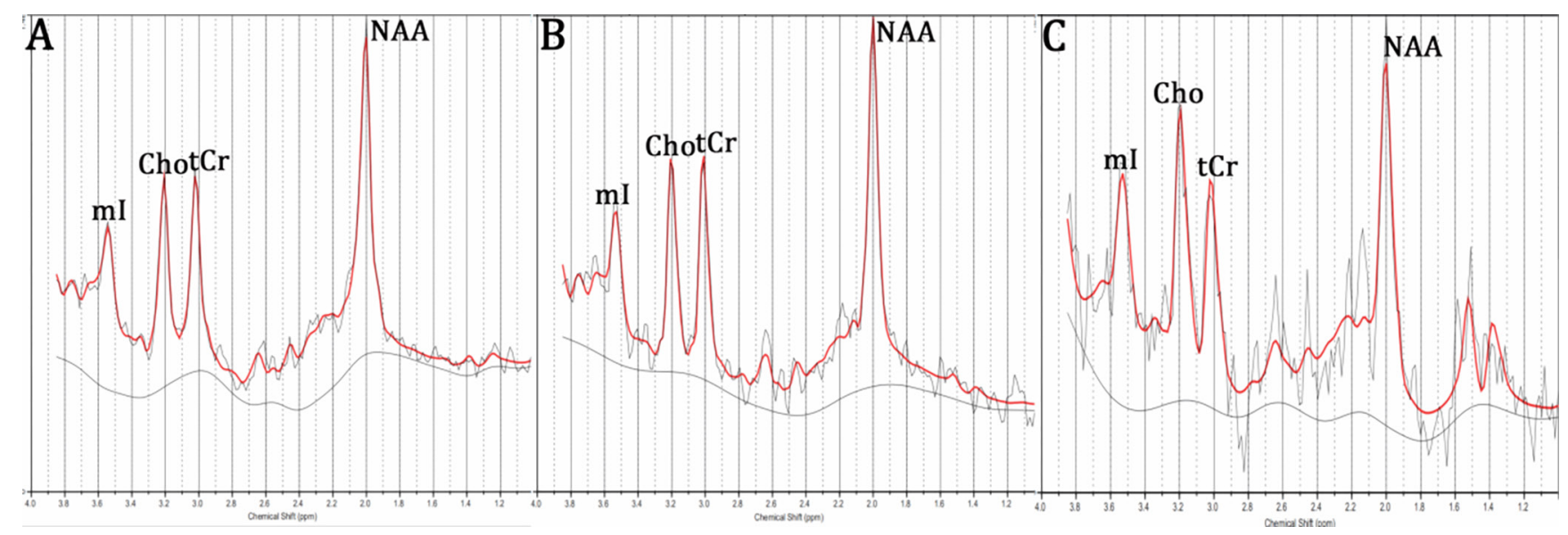

Figure S2 Examples of attained spectra from the right hippocampus. A = elderly controls, B = subjective cognitive decline plus, $\mathrm{C}=$ amnestic mild cognitive impairment. 
Supplementary material 6
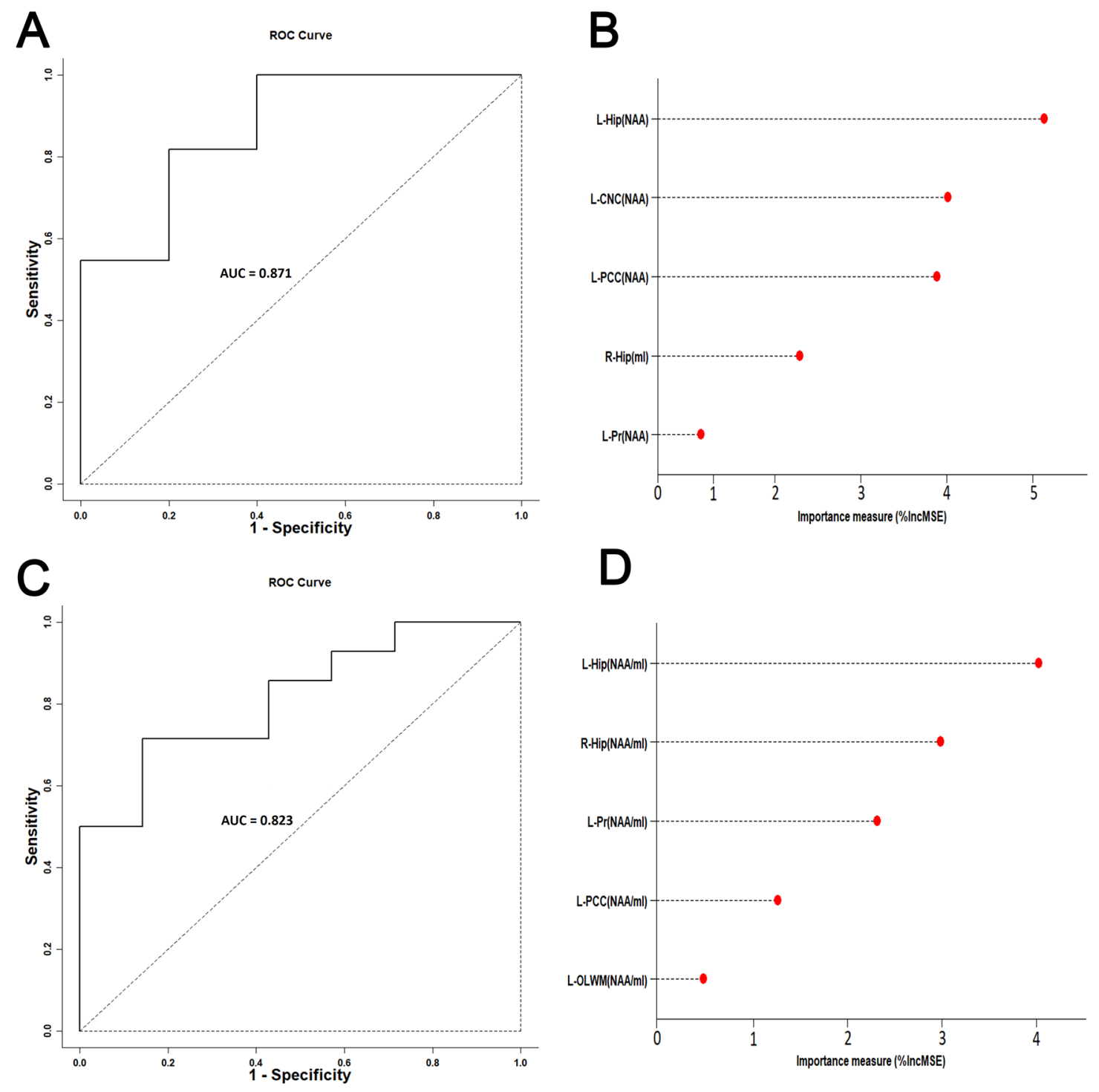

Figure S3 The AUC area and importance measurements from the random forest (RF) based on the testing dataset in discriminating participants with SCD plus from ECs. The horizontal and vertical coordinates represent specificity and sensitivity, respectively. A and B are from the RF in discriminating SCD plus from ECs using NAA and mI values. After variable selection, RF predicted with 0.871 accuracy; $\mathrm{C}$ and $\mathrm{D}$ are from the RF in discriminating SCD plus from ECs using NAA/mI ratio. After variable selection, RF predicted with 0.823 accuracy. Abbreviations: L-Hip = left hippocampus; R-Hip = right hippocampus; L-PCC = left posterior cingulate cortex; L-Pr = left precuneus; L-OLWM = left white matter of occipital lobe; L-CNC = left caput nuclei caudati; NAA = N-acetylaspartate; $\mathrm{mI}=$ myoinositol. 

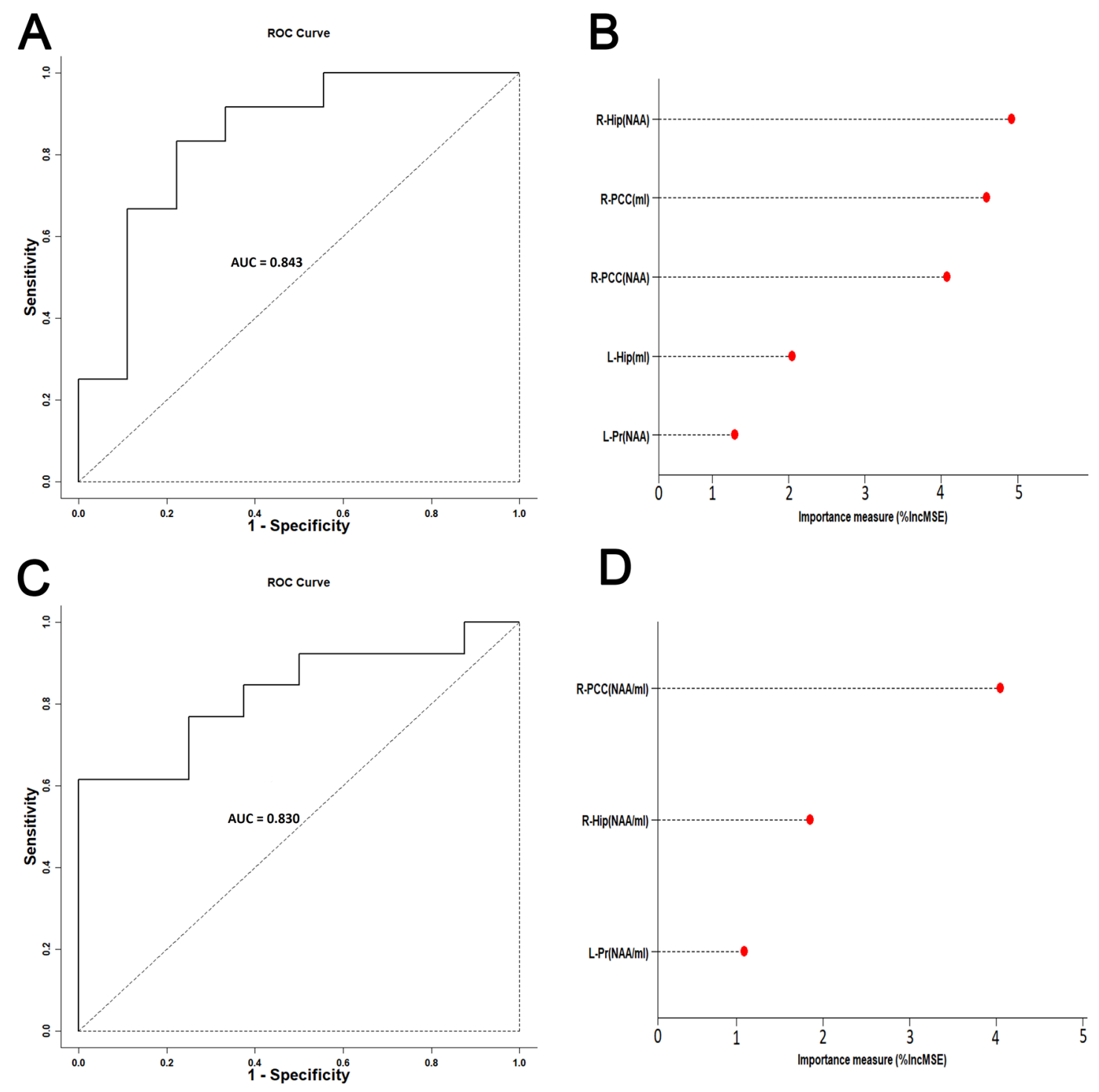

Figure S4 The AUC area and importance measurements from the random forest (RF) based on the testing dataset in discriminating participants with SCD plus from aMCI. The horizontal and vertical coordinates represent specificity and sensitivity, respectively. A and B are from the RF in discriminating SCD plus from aMCI using NAA and mI values. After variable selection, RF predicted with 0.843 accuracy; $\mathrm{C}$ and $\mathrm{D}$ are from the RF in discriminating SCD plus from aMCI using NAA/mI ratio. After variable selection, RF predicted with 0.830 accuracy. Abbreviations: L-Hip = left hippocampus; R-Hip = right hippocampus; L-Pr = left precuneus; R-PCC = right posterior cingulate; $\mathrm{NAA}=\mathrm{N}$-acetylaspartate $\mathrm{mI}=$ myoinositol. 


\section{Supplementary material 8}

Table S1 Group differences in VOI sizes and S/N in the left brain hemisphere of ECs, SCD plus, and aMCI participants

\begin{tabular}{|c|c|c|c|c|c|c|}
\hline VOls & Parameters & $E C(n=33)($ means $\pm S D)$ & SCD plus $(n=27)($ means $\pm S D)$ & aMCl $(n=31)$ (means \pm SD) & $\mathrm{F}$ & $P$ values \\
\hline \multirow[t]{2}{*}{ L-Hip } & VOI size $\left(\mathrm{cm}^{3}\right)$ & $3.202 \pm 0.468$ & $3.035 \pm 0.475$ & $2.997 \pm 0.385$ & 1.123 & 0.386 \\
\hline & $\mathrm{S} / \mathrm{N}$ & $7.112 \pm 1.108$ & $6.962 \pm 1.209$ & $6.826 \pm 1.369$ & 1.822 & 0.125 \\
\hline \multirow[t]{2}{*}{ L-PCC } & VOI size $\left(\mathrm{cm}^{3}\right)$ & $1.205 \pm 0.076$ & $1.186 \pm 0.080$ & $1.179 \pm 0.091$ & 1.231 & 0.322 \\
\hline & $\mathrm{S} / \mathrm{N}$ & $4.786 \pm 1.087$ & $4.658 \pm 1.169$ & $4.364 \pm 1.027$ & 0.664 & 0.738 \\
\hline L-Pr & $\mathrm{S} / \mathrm{N}$ & $4.845 \pm 1.100$ & $4.591 \pm 1.225$ & $4.532 \pm 1.132$ & 1.763 & 0.141 \\
\hline \multirow[t]{2}{*}{ L-OLWM } & VOI size $\left(\mathrm{cm}^{3}\right)$ & $2.392 \pm 0.261$ & $2.386 \pm 0.237$ & $2.375 \pm 0.227$ & 1.598 & 0.265 \\
\hline & $\mathrm{S} / \mathrm{N}$ & $5.852 \pm 1.209$ & $5.745 \pm 1.313$ & $5.585 \pm 1.367$ & 0.826 & 0.525 \\
\hline L-DT & VOI size $\left(\mathrm{cm}^{3}\right)$ & $3.576 \pm 0.531$ & $3.493 \pm 0.436$ & $3.489 \pm 0.391$ & 1.233 & 0.297 \\
\hline L-FLWM & $\mathrm{S} / \mathrm{N}$ & $4.602 \pm 1.219$ & $4.570 \pm 1.120$ & $4.346 \pm 1.109$ & 1.819 & 0.097 \\
\hline \multirow[t]{2}{*}{ L-LN } & VOI size $\left(\mathrm{cm}^{3}\right)$ & $1.199 \pm 0.096$ & $1.199 \pm 0.092$ & $1.194 \pm 0.081$ & 1.272 & 0.295 \\
\hline & $\mathrm{S} / \mathrm{N}$ & $4.349 \pm 1.551$ & $4.272 \pm 1.556$ & $4.016 \pm 1.655$ & 1.432 & 0.252 \\
\hline \multirow[t]{2}{*}{ L-CNC } & VOI size $\left(\mathrm{cm}^{3}\right)$ & $1.199 \pm 0.079$ & $1.183 \pm 0.077$ & $1.185 \pm 0.107$ & 1.316 & 0.300 \\
\hline & $\mathrm{S} / \mathrm{N}$ & $4.506 \pm 1.206$ & $4.307 \pm 1.462$ & $4.279 \pm 1.547$ & 1.462 & 0.281 \\
\hline
\end{tabular}

Abbreviations: ECs = elderly controls; SCD plus = subjective cognitive decline plus; $\mathrm{aMCl}=$ amnestic mild cognitive impairment; L-Hip = left hippocampus; L-PCC = left posterior cingulate cortex; L-Pr = left precuneus; L-DT = left dorsal thalamus; L-OLWM = left white matter of the occipital lobe; L-FLWM = left white matter of the frontal lobe; L-LN = left lenticular nucleus; L-CNC = left caput nuclei caudati; VOls $=$ volume of interests; $\mathrm{S} / \mathrm{N}=$ signal to noise. Values are presented as the mean $\pm \mathrm{SD}$. 


\section{Supplementary material 9}

Table S2 Group differences in VOI size $\left(\mathrm{cm}^{3}\right)$ and S/N in the right brain hemisphere of ECs, SCD plus, and aMCI participants.

\begin{tabular}{|c|c|c|c|c|c|c|}
\hline VOls & Parameters & $\mathrm{EC}(\mathrm{n}=33)$ (means $\pm S \mathrm{SD})$ & SCD plus $(n=27)($ means $\pm S D)$ & aMCl $(n=31)($ means $\pm S D)$ & $\mathrm{F}$ & $p$ values \\
\hline R-Hip & $\mathrm{S} / \mathrm{N}$ & $5.706 \pm 1.215$ & $5.962 \pm 1.209$ & $5.526 \pm 1.317$ & 1.452 & 0.263 \\
\hline \multirow[t]{2}{*}{ R-PCC } & VOI size $\left(\mathrm{cm}^{3}\right)$ & $1.188 \pm 0.084$ & $1.193 \pm 0.078$ & $1.785 \pm 0.103$ & 1.031 & 0.525 \\
\hline & $\mathrm{S} / \mathrm{N}$ & $4.686 \pm 1.047$ & $4.278 \pm 1.038$ & $4.658 \pm 1.122$ & 0.554 & 0.685 \\
\hline $\mathrm{R}-\mathrm{Pr}$ & $\mathrm{S} / \mathrm{N}$ & $4.872 \pm 1.134$ & $4.682 \pm 1.036$ & $4.553 \pm 1.152$ & 1.363 & 0.257 \\
\hline \multirow[t]{2}{*}{ R-OLWM } & VOI size $\left(\mathrm{cm}^{3}\right)$ & $2.383 \pm 0.261$ & $2.396 \pm 0.251$ & $2.378 \pm 0.239$ & 1.598 & 0.246 \\
\hline & $\mathrm{S} / \mathrm{N}$ & $5.852 \pm 1.321$ & $5.745 \pm 1.402$ & $5.292 \pm 1.567$ & 0.626 & 0.586 \\
\hline R-DT & VOI size $\left(\mathrm{cm}^{3}\right)$ & $2.593 \pm 0.527$ & $3.577 \pm 0.459$ & $3.563 \pm 0.472$ & 1.233 & 0.297 \\
\hline R-FLWM & $\mathrm{S} / \mathrm{N}$ & $4.711 \pm 1.308$ & $4.660 \pm 1.412$ & $4.523 \pm 1.314$ & 2.019 & 0.159 \\
\hline \multirow[t]{2}{*}{ R-LN } & VOI size $\left(\mathrm{cm}^{3}\right)$ & $1.205 \pm 0.101$ & $1.192 \pm 0.090$ & $1.184 \pm 0.107$ & 1.172 & 0.195 \\
\hline & $\mathrm{S} / \mathrm{N}$ & $4.398 \pm 1.651$ & $4.372 \pm 1.527$ & $4.335 \pm 1.625$ & 2.332 & 0.089 \\
\hline \multirow[t]{2}{*}{$\mathrm{R}-\mathrm{CNC}$} & VOI size $\left(\mathrm{cm}^{3}\right)$ & $1.196 \pm 0.094$ & $1.182 \pm 0.103$ & $1.881 \pm 0.095$ & 2.027 & 0.165 \\
\hline & $\mathrm{S} / \mathrm{N}$ & $4.676 \pm 1.512$ & $4.707 \pm 1.561$ & $4.856 \pm 1.397$ & 1.465 & 0.268 \\
\hline
\end{tabular}

Abbreviations: ECs = elderly controls; SCD plus = subjective cognitive decline plus; aMCI = amnestic mild cognitive impairment; R-Hip = right hippocampus; $\mathrm{R}-\mathrm{PCC}$ = right posterior cingulate cortex; $\mathrm{R}-\mathrm{Pr}=$ right precuneus; $\mathrm{R}-\mathrm{DT}$ = right dorsal thalamus; $\mathrm{R}-\mathrm{OLWM}=$ right white matter of the occipital lobe; R-FLWM = right white matter of the frontal lobe; R-LN = right lenticular nucleus; R-CNC = right caput nuclei caudati; $\mathrm{VOI}=$ volume of interest; $\mathrm{S} / \mathrm{N}=$ signal to noise. Values are presented as the mean $\pm \mathrm{SD}$. 